\title{
Is knowledge reliance in source guessing a cognitive trait? Examining stability across time and domain
}

\author{
Liliane Wulff ${ }^{1} \cdot$ Beatrice G. Kuhlmann ${ }^{1}$ \\ Published online: 7 January 2020 \\ (C) The Psychonomic Society, Inc. 2020
}

\begin{abstract}
When people need to infer the source of information in the absence of memory, they may rely on general knowledge (e.g., stereotypes) to guess the source. Prior research documented task-related determinants and individual differences of stereotype reliance in source guessing, but little is known about the underling nature of this process. In two experiments, we tested whether a cognitive trait could account for the knowledge reliance in source guessing. Participants performed two distinct study-test cycles of a classical source-monitoring paradigm in which two person sources present stereotypical information that in a later test phase had to be attributed to its origin. In Experiment 1, both tasks used item material from the same knowledge domain (age stereotypes) and were either separated by 10 minutes or 7 days. In Experiment 2, we used item material from two different knowledge domains (Task 1: age stereotypes; Task 2: gender stereotypes). Although cross-task correlations of source-guessing parameters from Bayesian-hierarchical multinomial processing tree model analyses showed only weak positive correlations, absolute source guessing remained fairly stable within individuals across time (Experiment 1) and knowledge domains (Experiment 2). Considering statistical challenges of the assessment of relative stability via correlations, we suggest based on the stricter absolute stability criterion that source guessing rather encompasses trait-like features. We discuss implications regarding the generalizability and nature of source guessing in comparison to other cognitive processes involved in source attribution, which were highly stable in both experiments.
\end{abstract}

Keywords Source guessing $\cdot$ Cognitive trait $\cdot$ Bayesian-hierarchical multinomial modeling

The ability to remember the source of information (e.g., who presented it) is crucial for many types of cognitive tasks in our everyday life (Johnson, Hashtroudi, \& Lindsay, 1993). For instance, we have to remember who told us something or where we read the latest news. The process of attributing information to sources is highly susceptible to prior knowledge, such as stereotypes and schemas (e.g., Bayen, Nakamura, Dupuis, \& Yang, 2000). For example, if we do not remember who told us about a new aerobic course in the gym, we may infer that the person source was a young adult rather than an old adult based on guessing in line with our stereotypes about

Electronic supplementary material The online version of this article (https://doi.org/10.3758/s13421-019-01008-1) contains supplementary material, which is available to authorized users.

Liliane Wulff

wulff@psychologie.uni-mannheim.de

1 Department of Psychology, School of Social Sciences, University of Mannheim, D-68131 Mannheim, Germany aging and what we typically associate with being young and old (Kuhlmann, Bayen, Meuser, \& Kornadt, 2016; Kuhlmann, Kornadt, Bayen, Meuser, \& Wulff, 2017). This inherent reliance on prior knowledge, of course, does not always lead to the correct source attribution and a biased misattribution could consequently support the maintenance (and reinforcement) of stereotypes over time.

Crucially, some studies have documented individual differences in the extent to which people rely on their prior knowledge in source attributions (Arnold, Bayen, Kuhlmann, \& Vaterrodt, 2013; Spaniol \& Bayen, 2002). Although influences on knowledge reliance in source guessing are well studied on the group level (contingency perception: e.g., Arnold et al., 2013; Bayen \& Kuhlmann, 2011; Kuhlmann, Vaterrodt, \& Bayen, 2012; Spaniol \& Bayen, 2002; source memory: e.g., Kuhlmann et al., 2016), little is known about the underlying mechanisms of source guessing that may explain the origin of individual differences. That is, why do some people strongly rely on their prior knowledge and others not at all? Is knowledge reliance in source attributions an intraindividual predisposition? Thus, the purpose of the current set of experiments 
was to examine individual knowledge-based source guessing and whether source guessing manifests a "cognitive trait" (Kantner \& Lindsay, 2012) — reflected in stability across time and (content) domain of the item material—or fluctuates across tasks, which in turn would imply that situational determinants of source guessing are primarily at play. Keeping in mind the detrimental effects of incorrect attributions of memories based on source guessing in the social environment (e.g., biased impression formation about others; Bell, Giang, Mund, \& Buchner, 2013; Ehrenberg \& Klauer, 2005; Sherman \& Bessenoff, 1999) or in the legal context (e.g., false accusations in court; Lindsay, 1994, 2014), it is essential to understand the potential persistence of such a source-guessing bias.

\section{Knowledge reliance in source guessing}

Attributing information to sources-defined as source monitoring - can be based on two cognitive processes: People can either rely on memory for contextual details (e.g., spatial, temporal, episodic, perceptual or affective details) or general knowledge (Johnson et al., 1993; Mitchell \& Johnson, 2000). General knowledge includes schemas that organize, link, and structure information based on previous experience (Alba \& Hasher, 1983), and stereotypes, "a set of beliefs about the personal attributes of a group of people" (Stroebe \& Insko, 1989, p. 5). The reliance on prior knowledge in source-monitoring tasks has been demonstrated in a multitude of studies (e.g., profession schemas: Bayen et al., 2000; room schemas: Küppers \& Bayen, 2014; gender stereotypes: Marsh, Cook, \& Hicks, 2006; social stereotypes: Ehrenberg \& Klauer, 2005; Sherman \& Bessenoff, 1999; age stereotypes: Kuhlmann et al., 2016).

Using Bayen et al.'s (2000) doctor-lawyer paradigm as an example, we will illustrate the standard experimental design for investigating influences of stereotypes and schemas on source monitoring. In the study phase, participants learn information presented by two sources (e.g., two persons: "Tom" $\&$ "Jim") that present an equal number of statements that are either typical for their source (i.e., consistent with the schema or stereotype associated with this source category; e.g., lawyer: "I have to be in court at nine") or statements that are typical for the other source (e.g., doctor: "It will take a couple of hours to get the results of this blood test"). At test, participants are informed about the specific category each source belongs to (e.g., profession of a doctor \& a lawyer), and they have to remember the source of each statement among new distractor statements.

In previous source-monitoring studies, participants commonly made more correct source attributions when a statement was typical for its source (e.g., Bayen et al., 2000). The cognitive processes that lead to this performance benefit for typical statements are not evident from the categorical responses given in the task at first sight. Both improved memory for or biased guessing in favor of typical statement-source combinations could explain the performance benefit. Multinomial processing tree (MPT) models such as the Twohigh-threshold multinomial model of source monitoring (2HTSM; Bayen, Murnane, \& Erdfelder, 1996) disentangle different cognitive processes contributing to observable behavior (e.g., Batchelder \& Riefer, 1999; Bayen et al., 1996). The 2HTSM decomposes the following processes from source-attribution behavior: item memory (i.e., the probability of recognizing a presented statement), source memory (i.e., the probability of remembering the source that presented a statement), item guessing (i.e., the probability of guessing that an unrecognized statement was old), and source guessing (i.e., the probability of guessing that a statement was presented by a specific [e.g., the typical] source).

Bayen et al. (2000) showed that schema-reliant source guessing, and not differential source memory, caused the performance benefit for typical statement-source pairs. ${ }^{1}$ When participants did not remember which source presented a statement, they guessed the schema-consistent source. Thus, a lack of memory is often found to be compensated with preexisting knowledge at least when analyzing the data on the group-level (i.e., aggregated across participants; Arnold et al., 2013; Bayen \& Kuhlmann, 2011; Bayen et al., 2000; Ehrenberg \& Klauer, 2005; Kuhlmann et al., 2016; Kuhlmann et al., 2012; Küppers \& Bayen, 2014; Spaniol \& Bayen, 2002). As MPT models are merely measurement models that quantify certain cognitive processes, the question what determines these processes - and especially biased source guessing - remains open. Several determinants of biased source guessing have been identified already: misperception of item-source contingency (Arnold et al., 2013; Bayen \& Kuhlmann, 2011; Kuhlmann et al., 2012; Spaniol \& Bayen, 2002), poor source memory (Arnold et al., 2013; Kuhlmann et al., 2016), reduced cognitive capacities at encoding (Bayen \& Kuhlmann, 2011; Ehrenberg \& Klauer, 2005; Kuhlmann et al., 2012), and provision of category information about sources after encoding (Hicks \& Cockman, 2003; Kuhlmann et al., 2012). Even though these determinants can explain differences in source guessing between experimental conditions, they do not consider remaining interindividual variance (but see Arnold et al., 2013; Spaniol \& Bayen, 2002) and whether biased guessing happens occasionally or consistently.

\footnotetext{
${ }^{1}$ Schemas/stereotypes can also influence source memory. If an item is very atypical for the source with which it was presented, then attention is drawn to this expectation-violating item-source combination, which may result in better source memory for the atypical, schema-incongruent or stereotypeincongruent information (i.e., inconsistency effect; e.g., Bell, Buchner, Kroneisen, \& Giang, 2012; Ehrenberg \& Klauer, 2005; Kroneisen \& Bell, 2013; Küppers \& Bayen, 2014). However, this is not consistently observed whereas schema-based/stereotype-based source guessing is consistently observed, even when this inconsistency effect occurs. In any case, this guessing bias leads to a performance advantage for typical item-source combinations.
} 
Recent methodological advances in the estimation of MPT model parameters offer more sophisticated statistical analyses and allow for inferences about cognitive processes on an individual level. One such advance is the latent-trait model, a Bayesian-hierarchical extension of multinomial models proposed by Klauer (2010). The latent-trait model accounts for variability between individuals and estimates parameters that reflect latent cognitive processes for each individual separately. With this extension, individual parameter estimates can be compared and correlated across different applications of a task (e.g., across time and stimulus material).

\section{Cognitive trait}

One possible extension to the aforementioned group-level determinants could be that individual differences in knowledge reliance in source guessing reflect trait-like stability. Independent of the experimental condition, some people might be more inclined to rely on knowledge to fill memory gaps whereas others might be less inclined to do so. If one follows the definition of Roberts (2009), trait-like stability is "meaningfully consistent" ( $p .139)$ behavior. That is, although behavior should be consistent across certain situations it must not be identical in order to be considered stable. Trait-like stability would, for instance, be reflected in correlational evidence of two variables but not necessarily in the same numeric point estimates across situations. For response tendencies such as old-new guessing bias in recognition tasks (Kantner \& Lindsay, 2012, 2014), knowledge reliance in recognition heuristic (Michalkiewicz \& Erdfelder, 2016), and risky decisionmaking (Glöckner \& Pachur, 2012), trait-like stability in these cognitive processes underlying responses was reported.

In a study by Kantner and Lindsay (2012), individuals learned English nouns for a later memory test in two separate study-test cycles. After studying these items, individuals indicated whether they had learned the items before or not. The tendency to respond that a previously studied item was presented ("old"), measured as signal-detection theory (SDT) response-bias measure $c$ (Macmillan, 1993), correlated highly across tests, separated by either 10 minutes or seven days, and also across stimulus material (words \& digital images of paintings). The authors concluded that the old-new response bias in memory is a cognitive trait that should not vary deliberately within individuals and has predictive value given comparable experimental settings across tests. They explicitly distinguished it from a personality trait but acknowledged that cognitive and personality traits could be associated.

Cross-task stability of the reliance on prior knowledge has also been documented for the recognition-heuristic use in judgment tasks (i.e., choosing the recognized object and ignore knowledge about it; Goldstein \& Gigerenzer, 2002). Applying a latent-trait MPT model, Michalkiewicz and Erdfelder (2016) found strong correlations of individuals' recognition-heuristic use between two tests, separated by either one or seven days or immediately succeeding each other but with varying judgmentcontent domains. Thus, the general tendency to rely on the recognition heuristic (i.e., on one's prior knowledge) in judgments seems to be a cognitive disposition that is stable across time and independent of the knowledge domain.

Even though the source-guessing bias is somewhat different from the old-new response bias (Kantner \& Lindsay, 2012) and the recognition-heuristic use (Michalkiewicz \& Erdfelder, 2016), these measures share not only task-related features such as the mechanism of recognition, but, especially, the recognition-heuristic use shares a common content feature with source guessing: people's reliance on prior knowledge. Thus, the trait-like stability may also hold for knowledgebased source guessing but has not been tested yet.

\section{Cognitive trait $=$ personality trait?}

Kantner and Lindsay (2014) examined "personality trait-like qualities" ( $p .1273)$, defined as an association between a response bias and a personality trait (a detailed description of key personality traits and cognitive-processing styles discussed in this section can be retrieved from Table 1). Their results brought only weak evidence for a correlation with need for cognition (NFC; Cacioppo \& Petty, 1982) and internal punishment-reward preference (behavioral inhibition system/behavioral activation system; Carver \& White, 1994) scores. Whereas a cognitive trait may be independent from a personality trait, Kantner and Lindsay (2014) acknowledged that this association may nonetheless exist. Michalkiewicz, Minich, and Erdfelder (2019) assessed the relation between the recognition-heuristic use and NFC as well as faith in intuition (FII; Epstein, Pacini, Denes-Raj, \& Heier, 1996). They found a negative correlation of recognition-heuristic use and NFC but no substantial correlation with FII. Participants who scored low on NFC tended to use the recognition heuristic more frequently. This relationship was unique to NFC and held even after controlling for the Big Five personality traits. So, the recognition-heuristic use possesses personality traitlike qualities at least to some extent.

If knowledge reliance in source guessing turns out to be a cognitive trait, personality traits may be related to it as well. Additionally, knowledge reliance in source guessing may be also related to stereotypical thinking in general. We often use stereotypes as a cognitive tool (Gilbert \& Hixon, 1991; Macrae, Milne, $\&$ Bodenhausen, 1994) to evaluate members of social groups because individuating is mentally effortful (Fiske, 1989). This lack of motivation to process individual information deeply could also hold for knowledge-based source guessing. For instance, and in line with the aforementioned research on the recognition heuristic, stereotyping is related to NFC in some studies. That is, people who score low on NFC tend to use stereotyping as mental shortcuts more often (e.g., Carter, Hall, Carney, \& Rosip, 2006; 
Table 1 Definition of exemplar (personality trait) constructs related to knowledge reliance and response bias

\begin{tabular}{|c|c|c|}
\hline Construct & Definition & Reference \\
\hline Recognition heuristic (RH) & $\begin{array}{l}\text { Simple decision strategy applied in dichotomous judgment tasks. For } \\
\text { instance, when participants need to answer the question "Which city is } \\
\text { more populous: Tokyo or Busan?," they should choose the recognized } \\
\text { object (here: Tokyo) according to the RH because they immediately } \\
\text { recognize this city (and not the other; here: Busan). They could integrate } \\
\text { more detailed knowledge about the city (e.g., that cities with international } \\
\text { airports such as Tokyo are often populous) and come to the same choice. }\end{array}$ & $\begin{array}{l}\text { e.g., Goldstein \& Gigerenzer (2002); } \\
\text { cf. Michalkiewicz \& } \\
\text { Erdfelder (2016) }\end{array}$ \\
\hline Need for cognition (NFC) & $\begin{array}{l}\text { Individual's tendency to engage in effortful, deep thinking and to enjoy } \\
\text { structuring situations in a meaningful way. }\end{array}$ & cf. Cacioppo \& Petty (1982) \\
\hline Faith in intuition (FII) & $\begin{array}{l}\text { Individual's tendency to rely on intuitive, experiential processing of } \\
\text { information. }\end{array}$ & $\begin{array}{l}\text { cf. Epstein, Pacini, Denes-Raj, } \\
\text { \& Heier (1996) }\end{array}$ \\
\hline Big Five & $\begin{array}{l}\text { Taxonomy for five basal personality traits. Openness for Experience } \\
\text { contrasts curious and exploratory trait facets with rigid and traditional } \\
\text { ones. Conscientiousness contrasts disciplined trait facets with } \\
\text { unambitious ones. Extraversion contrasts warm and outgoing trait facets } \\
\text { with reserved ones. Agreeableness contrasts generous and honest trait } \\
\text { facets with selfish and aggressive ones. Neuroticism contrasts calm and } \\
\text { stable trait facets with sad and scared ones. }\end{array}$ & $\begin{array}{l}\text { e.g., Goldberg (1993); John \& } \\
\text { Srivastava (1999); cf. McCrae \& } \\
\text { Costa (2008) }\end{array}$ \\
\hline $\begin{array}{l}\text { Behavioral inhibition } \\
\text { system/behavioral activation } \\
\text { system (BIS/BAS) }\end{array}$ & $\begin{array}{l}\text { Taxonomy for two motivational systems that underlie negative and positive } \\
\text { affect. The BIS reflects an orientation towards aversive outcomes; the } \\
\text { BAS reflects an orientation towards pleasant outcomes. The BAS scale } \\
\text { can be subdivided into reward responsiveness, drive, and fun seeking. }\end{array}$ & cf. Carver \& White (1994) \\
\hline
\end{tabular}

Note. Description of central concepts mentioned in the introductory paragraph including examples where these concepts have been referred to in the literature

Perlini \& Hansen, 2001) or even less often in terms of stereotypeconsistent recall (Crawford \& Skowronski, 1998). Transferred to source guessing, this mixed evidence in terms of the direction of relation suggests that source guessing and NFC could be related, although the direction of correlation cannot be derived. With regard to the Big Five personality traits, Flynn (2005) showed that the trait Openness to Experience was negatively correlated with explicit interracial attitudes and positively with impressions of other-race persons. Carter et al., (2006) showed that the willingness to accept stereotyping is negatively correlated with Agreeableness and positively with Extraversion and Neuroticism. Assuming that the knowledge reliance in source guessing is also used to form an impression about other persons, one could speculate that it may be negatively correlated with Openness to Experience and Agreeableness and positively correlated with Extraversion and Neuroticism (based on Carter et al., 2006; Flynn, 2005). It is, however, generally debatable whether the correlation of stereotyping with personality traits or cognitive-processing styles can be transferred to stereotyping in memory tasks, and more specifically, to the cognitive process of source guessing, and this thereof remains an exploratory research question.

\section{Overview of the current experiments}

We knew from prior research that people differ in the extent to which they make use of stereotypes/schemas in source guessing (e.g., Arnold et al., 2013; Spaniol \& Bayen, 2002). In two experiments, we examined the stability of knowledge reliance in source guessing to test whether Kantner and Lindsay's $(2012,2014)$ findings characterizing response bias as a cognitive trait generalize above and beyond old-new item guessing and the methodological approach of signal-detection theory to multinomial processing tree modeling. Following Michalkiewicz and Erdfelder (2016), we tested two different facets of stability as a trait-like predisposition-stability across time and knowledge domain. Therefore, we applied two distinct source-monitoring tasks to each participant, using item material of the same stereotype domain but separated by a time interval of 10 minutes or seven days (Experiment 1) or different stereotype material between tasks (Experiment 2).

Other than Kantner and Lindsay $(2012,2014)$, we specified a Bayesian-hierarchical model version of the 2HTSM (Bayen et al., 1996) to assess parameter correlations, but our data did nonetheless also allow for a replication of their findings with both MPT and SDT measures of response bias. In addition to this relative stability, we examined the absolute stability measured as the absolute difference of source guessing between tasks. Based on Roberts's (2009) stability definition, we did not necessarily expect to observe the exact same point estimates of source guessing across tasks (i.e., absolute stability) but rather a correlation across time or knowledge domain irrespective of the overall group-level estimate (i.e., relative stability). Participants who guess stereotype based more strongly than others in one test should be more prone to do so in another test even if the mean group-level source guessing 
changes (e.g., due to regression to the mean or reactive effects from the first task).

\section{Experiment 1: Stability across time}

If stereotype-based source guessing is a cognitive trait, it should be stable within individuals across time (Kantner \& Lindsay, 2012; Roberts, 2009). Therefore, we tested whether participants show comparable (absolute and/or relative to the group level) knowledge reliance in source guessing in two separate source-monitoring tasks, performed either 10 minutes or seven days apart. We estimated individual sourcemonitoring parameters and cross-task correlations/ differences using Bayesian-hierarchical modeling (latent trait; Klauer, 2010).

\section{Method}

\section{Participants and design}

The design was a 2 (time interval between tasks; between subjects) $\times 2$ (source age: old vs. young; within subjects) $\times$ 2 (age stereotypicality: statement typicality for respective source age; within subjects) mixed factorial. As there is currently no appropriate power analysis for Bayesian-hierarchical MPT models available, we computed the equivalent frequentist analysis for orientation. An a priori power analysis in G*Power 3 (Faul, Erdfelder, Lang, \& Buchner, 2007) revealed that assuming a moderate positive correlation of source guessing across time, Pearson's $r=.30, n=82$ participants per condition are needed to yield a power $(1-\beta)$ of .80 at $\alpha=.05$ (two-tailed). We increased the number of participants per condition beyond a minimum of 100 for two reasons: first, to increase estimation precision and, second, to fulfill our counterbalancing constraints. Additionally, we conducted recovery simulations to test the precision of parameter estimation for the recruited sample size and used item number in the experiment (see Table S1 in the Supplemental Material). ${ }^{2}$

In total, 224 students of the Universities of Mannheim and Heidelberg participated for psychology course credit or monetary compensation. ${ }^{3}$ We randomly assigned participants to two conditions (between subjects) in which they had to complete two age-stereotype source-monitoring tasks either

\footnotetext{
${ }^{2}$ We thank an anonymous reviewer for suggesting these parameter-recovery simulations.

${ }^{3}$ For unknown reasons, source-guessing variance was strongly restricted for the first 54 participants in the 7-day condition, preventing a meaningful correlation analysis (see Fig. S1 in the Supplemental Material). Therefore, we started data collection for this condition anew. Analyses based on the initial data set of 54 participants can be retrieved from the Supplemental Material as well as analyses based on the initial 54 (see Fig. S1) and additional 110 participants combined (see Fig. S2). Inclusion of these data did not change the conclusions (see Tables S2 and S3).
}

separated by 10 minutes $(n=114 ; M=21.37$ years, $S D=$ 2.11 years, age range: $18-26$ years, $76 \%$ women, $40 \%$ psychology majors) or seven days ( $n=110 ; M=20.76$ years, $S D$ $=2.12$ years, age range: $18-26$ years, $82 \%$ women, $50 \%$ psychology majors). Exclusion criteria during participant recruitment were color-blindness, age $>26$ years (i.e., older than the younger participants in the survey study for norming the item material; see Material section), neurological disorders, previous participation in a similar experiment, and insufficient German proficiency (i.e., learned after the age of 6).

\section{Material}

Age-stereotypical statements for the source-monitoring tasks consisted of everyday statements selected from a previous survey study (Kuhlmann et al., 2017). In this survey, 74 older ( $M=70.17$ years, $60-84$ years) and 69 younger $(M=22.03$ years, $18-26$ years) participants rated the age typicality of 368 statements reflecting the positive or negative pole of three adjective dimensions (autonomy, instrumentality, and integrity) in five life domains (family \& partnership; finances; friends \& acquaintances; health, fitness, \& appearance; religion \& spirituality). An example statement is: "I volunteer at church." (adjective dimension: instrumentality [positive]; domain: religion \& spirituality). The typicality of a statement for either a "young adult" or an "old adult" (between subjects) was rated on a 5-point Likert scale $(1=$ very atypical, $2=$ atypical, $3=$ neither typical nor atypical, $4=$ typical, $5=$ very typical). Following Kuhlmann et al. (2016), we defined a statement as typically-old if the mean typicality rating for an "old adult" was $\geq 3$ and, at the same time, $<3$ for a "young adult" and vice versa for typically-young statements. One hundred and thirteen typically-old and 103 typically-young statements fit our criterion. Out of these, we randomly selected 60 typically-old and typically-young statements each and divided them into two item sets of 60 statements ( 30 of each typicality) for the two tasks. Item sets were comparable in their mean typicality ratings (all $p \mathrm{~s} \geq .649$ ), polarity, adjective dimension and life domain. Furthermore, each of the 60-item sets was divided into three matched subsets of 20 statements, of which two subsets served as study lists and one as distractors at test. The assignment of item lists to study and distractor lists was counterbalanced across participants. ${ }^{4}$ Further, the source ages and names were counterbalanced across tasks. At test, one source's age was indicated to be 70 years and the other's to be 23 years because these were the ages participants thought of while rating the statement typicality for an "old adult" or a "young adult," respectively, in the

\footnotetext{
${ }^{4}$ The assignment of study and distractor lists was not fully counterbalanced, as for organizational reasons more participants took part than necessary to fulfill counterbalance constraints. Because the differences in the number of participants between counterbalance conditions were small and not at all systematic, we preferred to analyze all collected data.
} 
survey (Kuhlmann et al., 2017). In both tasks, common German last names (without indicating age or gender) served as sources: either "Müller" and "Schneider" or "Fischer" and "Schmidt."

Both experiments included the BFI-2 short form (Soto \& John, 2017) derived from the German self-report long form (Danner et al., 2016; Rammstedt, Danner, Soto, \& John, 2018). This short form consists of 30 items assessing the Big Five personality traits under 15 specific facet traits (each two items). ${ }^{5}$ Items were rated on a 5-point Likert scale ( 1 = strongly disagree, 5 = strongly agree). Need for cognition (NFC) and faith in intuition (FII) were assessed with the German translation of the Rational Experiential Inventory (REI; Epstein et al., 1996; Keller, Bohner, \& Erb, 2000). The REI consists of 29 items, 14 assessing the construct of NFC, 15 assessing FII, rated on a 7 -point Likert scale $(1=$ completely false, $7=$ completely true).

\section{Procedure}

Experiments were programmed with OpenSesame (Mathôt, Schreij, \& Theeuwes, 2012) and run on computers with a screen resolution of $1,280 \times 1,024$ pixels. Participants were tested in groups up to four in individual computer booths. After providing written informed consent, each participant performed two distinct study-test cycles of a sourcemonitoring task. In each task, participants learned information presented by two sources that they had to remember in a following test phase. To render both tasks comparable, participants were explicitly instructed before learning that both item and source memory would later be tested. Although incidental source learning results in greater source-guessing bias (Kuhlmann et al., 2012), we used intentional source-learning instructions for both tests as participants would have known that we test their source memory during the second task based on the first tasks' memory test. We further informed participants that one source person was old and the other young but did not reveal the specific ages until test. We presented the statements trial by trial in the study phase, each combined with one of two source names. Each source presented half typical information and half atypical information. For instance, the "old adult" was associated with typically-old behavior, such as going to church or visiting the doctor but, at the same time, with typically-young behavior, such as going to the gym and meeting friends for a shopping tour, equally often. The source names were printed in green or yellow (counterbalanced between subjects) on black background (font: sans; font size: $35 \mathrm{pt}$ ); the statements were written in white below (font: sans; font size: $32 \mathrm{pt}$ ). Each statement-source combination remained

\footnotetext{
${ }^{5}$ Due to experimenter error, the first 26 questionnaires lacked one item for the domain of conscientiousness (from the trait facet responsibility), which we thus dropped from analyses for all participants in Experiment 1. We used the corrected questionnaire in Experiment 2.
}

on the screen for $4 \mathrm{~s}$ followed by an interstimulus interval of $250 \mathrm{~ms}$. We randomized the order of statement-source combinations restricted to three subsequent statements presented by the same source.

At test, we informed participants that it might be helpful to know social category information about the sources (i.e., which source was 70 vs. 23 years old) for the upcoming memory test. During the self-paced test phase, participants again saw all statements from the study phase randomly intermixed with one-third new distractor statements. Participants then decided for each statement whether it had been presented with one of the two sources or was new. We presented the question "Who said" written in white font in the top left corner of the screen, and the test statement, centered on the screen. In the center, one source name was presented to the left, the other source name was presented to the right, both in the corresponding color (green or yellow) as in the study phase with the age information below (i.e., "70 years" vs. "23 years"). We additionally presented the response option "NEW" in the bottom center in red font. Participants responded via pressing the key " $D$ " for the left response option, " $K$ " for the right response option, and the space bar for the new response option. Key positions on the used QWERTZ keyboard corresponded to source position on the screen and were additionally marked with colored (green, yellow, red) stickers. Finally, participants estimated their perceived ratio of age-typical statementsource combinations (contingency judgment). That is, they estimated how many of the statements from the study phase of each typicality category (i.e., young, old; order counterbalanced between tasks and within subjects) had been presented by each source. The procedure for the second source-monitoring task was equivalent to the first but used different source labels (e.g., first task: "Fischer" \& "Schneider"; second task: "Müller" \& "Schmidt") and agestereotypical statements.

We randomly assigned participants to either complete the two tasks in the first session, separated by 10 minutes, or one per session, separated by seven days. In the first session, participants in the 10-minute condition worked on the first source-monitoring task, filled out the demographic questionnaire and the BFI-2 within the fixed interval of 10 minutes (measured with a stopwatch) followed by the second sourcemonitoring task. If participants finished the questionnaires in less than 10 minutes, they were instructed to wait quietly until time had passed (nobody needed more than 10 minutes). Participants in the 7-day condition completed the first source-monitoring task and then rated the typicality of statements for either a man or a woman (used in Experiment 2) and completed the REI. Both participant groups returned to the lab one week later on the same day and, if possible, at the same time as for the first appointment ( \pm 8 hours) to ensure comparability in the procedures. Participants in the 10-minute condition now rated the statement's gender typicality and filled 
out the REI; participants in the 7-day condition worked on the second source-monitoring task, then filled out the demographic questionnaire and the BFI-2. Finally, all participants were debriefed and compensated.

\section{Results and discussion}

\section{Model-based analyses}

We applied the 2HTSM (Bayen et al., 1996) to estimate the following underlying cognitive processes based on the observed response frequencies collected in each sourcemonitoring task: The probability of item recognition is measured by parameter $D$, separately for statements that were originally presented by the typical source $\left(D_{\mathrm{T}}\right)$, the atypical source $\left(D_{\mathrm{A}}\right)$, or are new $\left(D_{\mathrm{N}}\right)$. When a statement is recognized, the source may also be remembered with probability $d$. More specifically in the present paradigm, with probability $d_{\mathrm{T}}$ the typical source is remembered, whereas with $d_{\mathrm{A}}$ the atypical source is remembered. When source memory fails (with probability $1-d_{\mathrm{T}}$ or $1-d_{\mathrm{A}}$, respectively), guessing processes are engaged. Specifically, parameter $a$ measures the probability to guess that a recognized statement is presented by the typical source and with the complementary probability $1-a$ that the statement is presented by the atypical source. If item memory fails, the source can also not be remembered (Bell, Mieth, \& Buchner, 2017; Malejka \& Bröder, 2016; see also Klauer \& Kellen, 2010). With probability $b$, participants then guess that a statement was previously presented in the study phase (i.e., is "old"), either followed by guessing the typical (probability $g$ ) or atypical source (probability $1-g$ ). With probability $1-$ $b$, participants guess that the statement is new.

We used Submodel 4 of the 2HTSM (cf. Bayen et al., 2000) which has been used to analyze data from similar paradigms in prior studies (Kuhlmann et al., 2016; Spaniol \& Bayen, 2002), illustrated in Fig. 1. This is the most parsimonious model to start with as it explains source-monitoring data with four parameters only: $D$ measures the probability of recognizing a statement as old, regardless of the originally presenting source, or new (i.e., $D_{\mathrm{T}}=D_{\mathrm{A}}=D_{\mathrm{N}}=D$ ). Parameter $d$ measures the probability of remembering the source of a recognized old statement (i.e., $d_{\mathrm{T}}=d_{\mathrm{A}}=d$ ). Parameter $b$ measures the probability of guessing "old." And, crucially, $g$ measures the probability of guessing the typical source, regardless of whether the statement was recognized or merely guessed to be old (i.e., $a=g$ ). Thus, the data basis for our analysis consisted of "typical" trials (aggregating attributions across typically-old statements presented by the "old" source \& typically-young statements presented by the "young" source), "atypical" trials (aggregating attributions across typically-old statements presented by the "young" source \& typicallyyoung statements presented by the "old" source) and new trials (aggregating attributions across typically-young and old distractors). ${ }^{6}$

To obtain individual parameter estimates and correlations, we applied a latent-trait Bayesian-hierarchical extension of multinomial modeling (Klauer, 2010). This approach accounts for variability between participants by treating parameters as random variables. ${ }^{7}$ Individual model parameters and their correlations are estimated jointly in one model. Separate parameter estimates for each person are constrained by the population-level model that assumes a multivariate normal distribution of the probittransformed model parameters with a mean and covariance matrix to be estimated from the data. This Bayesian-hierarchical approach is particularly advantageous here because, in addition, it allows inclusion of external covariates of model parameters (e.g., personality traits). Samples from the posterior distribution of parameters are drawn with the Monte Carlo-Marcov chain (MCMC) algorithm. We used the $R$ package TreeBUGS for this purpose (Heck, Arnold, \& Arnold, 2018). To fit a latent-trait MPT model, prior distributions for the group-level mean and covariance are needed that are updated by the incorporated data resulting in a posterior distribution of model parameters. Following Klauer (2010) and Matzke, Dolan, Batchelder, and Wagenmakers (2015), TreeBUGS uses weakly informative priors (see the Supplemental Material or Heck et al., 2018, for further details). The number of iterations for each model was fit to convergence. That is, we cycled through three MCMC chains with 20,000 iterations each until all parameters reached the desired convergence criterion as indicated by $\hat{R}<1.05$ (Gelman \& Rubin, 1992). In order to check for convergence stability, we ran

\footnotetext{
${ }^{6}$ Following a suggestion by an anonymous reviewer, we additionally fitted an alternative no-bias model attributing any typicality-based differences in source attributions to differences in source memory (i.e., $d_{\mathrm{T}}$ versus $d_{\mathrm{A}}$ estimated independently) but not source-guessing bias (i.e., $a=g=.50$; retaining the item-memory restriction $D_{\mathrm{T}}=D_{\mathrm{A}}=D_{\mathrm{N}}$ ). This model neither fit the data from the 10-minute condition of Experiment $1, T_{1}<.001, T_{2}<.001$, nor the data from Experiment 2, $T_{1}=.001, T_{2}<.001$. In the 7-day condition, this no-bias model merely fit in the mean structure, $T_{1}=.09$, but not in the covariance structure $T_{2}<.001$. The trade-off between model fit and complexity using the Bayesian measure DIC (Spiegelhalter, Best, Carlin, \& Van der Linde, 2002) revealed that Submodel 4 (reported in the main analysis of the manuscript; equating source memory for typical and atypical statement-source combinations) is preferred across all experiments/conditions (10-minute condition of Experiment 1, DIC $\mathrm{D}_{\text {Submodel } 4}$ : 4718 vs. $\mathrm{DIC}_{\mathrm{No} \text {-bias Model }}$ 4939; 7-day condition of Experiment 1, DIC $\mathrm{Submodel}_{\text {4: }} 4541$ vs. DIC No-bias Model $_{4}$ 4656; Experiment 2, $\mathrm{DIC}_{\text {Submodel 4}}$ : 5125 vs. DIC No-bias Model 5528 ). This confirms that the typicality effects we observed in source attributions are best described by sourceguessing biases, not source-memory differences. We thus retained Submodel 4 of the 2HTSM for the main analyses as this was the most parsimonious model, which fit the data from all our experimental conditions well and provides a valid measure of source-guessing bias.

${ }^{7}$ Traditionally, MPT model parameters are estimated from the group-level aggregated response frequencies using maximum likelihood estimation (e.g., Hu \& Batchelder, 1994), and thus inherently assuming homogeneity of both participants and items (Batchelder \& Riefer, 1999). As a result, MPT models can be misspecified and their parameter estimates biased if participants and/or items are heterogeneous (Klauer, 2010; Matzke, Dolan, Batchelder, \& Wagenmakers, 2015; Smith \& Batchelder, 2010). Most crucially, individual differences are ignored in this analysis approach.
} 


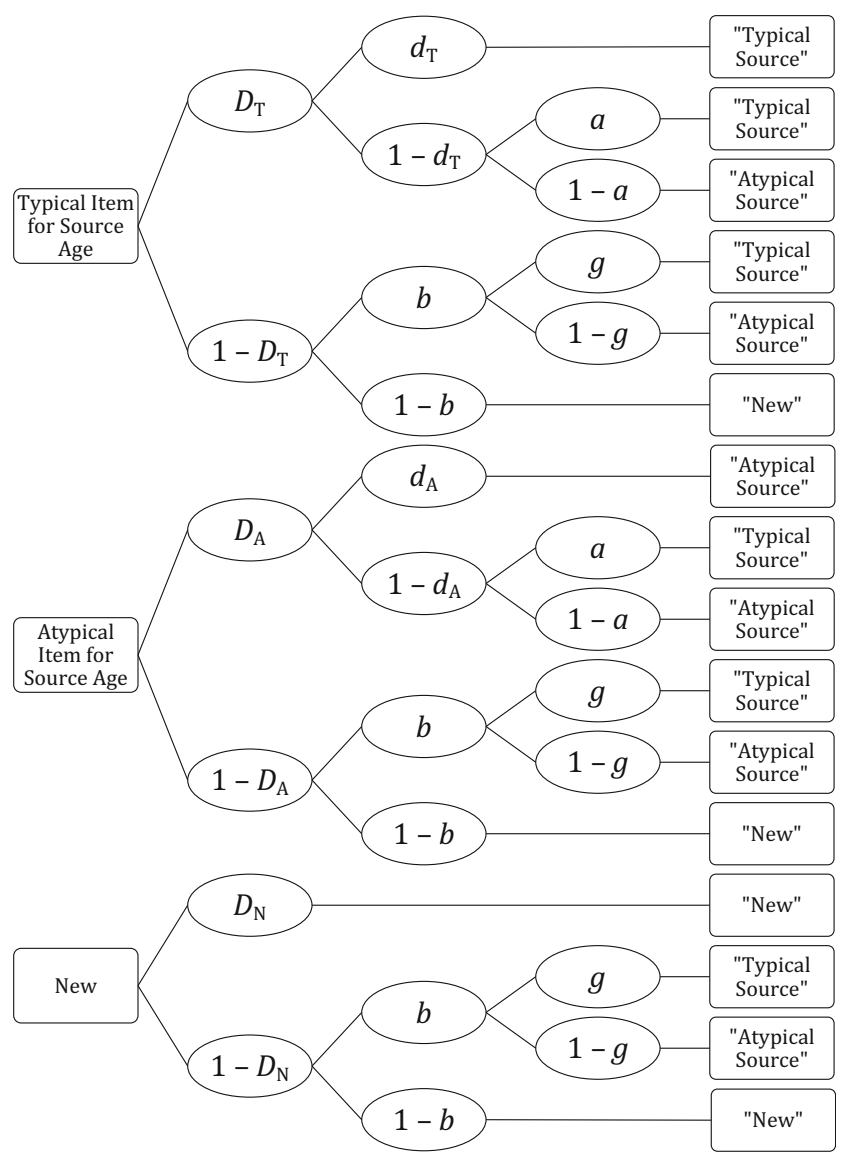

Fig. 1 Two-high-threshold multinomial model of source monitoring adapted to the current paradigm. $D_{\mathrm{T}}=$ probability of recognizing a statement that had been presented by a typical source; $D_{\mathrm{A}}=$ probability of recognizing a statement that had been presented by an atypical source; $D_{\mathrm{N}}=$ probability of knowing a statement is new; $d_{\mathrm{T}}=$ probability of correctly remembering the source of a statement that had been presented with a typical source; $d_{\mathrm{A}}=$ probability of correctly remembering the source of a statement that had been presented with an atypical source; $b=$ probability of guessing that an unrecognized statement is old; $a=$ probability of guessing that a recognized statement had been presented by the typical source; $g$ = probability of guessing that an unrecognized statement had been presented by a typical source. Adapted from "Source Discrimination, Item Detection, and Multinomial Models of Source Monitoring" (Bayen et al., 1996)

one additional cycle of 20,000 iterations. The convergence criterion was maintained for this additional cycle in all our analyses; otherwise, the estimation would have started anew.

We tested whether the model adequately describes our data using the test statistics $T_{1}$ and $T_{2}$ proposed by Klauer (2010). $T_{1}$ tests the mean fit and $T_{2}$ the covariance fit by computing the distance between expected and observed mean frequencies or covariances, respectively, based on Pearson's $\chi^{2}$ statistic. A satisfactory group-level model fit is indicated by a posterior predictive $p$ value $>.05$. We obtained good model fit for the means $T_{1}$ in both conditions, $p=.111$ (10 minutes) and $p=.29$ (7 days) but observed a satisfactory model fit for the covariance structure only in the 7-day condition, $p=.062$, and not in the 10-minute condition, $p=0 .^{8}$

We report the group-level mean estimates of the posterior distribution for the four source-monitoring parameters and corresponding $95 \%$ Bayesian credibility intervals $(\mathrm{BCI})$ as presented in Table 2. In the following, we will focus on the sourceguessing parameter $g$. We first examined whether there was a stereotype bias in source guessing on the group level. Therefore, we sampled the posterior distribution for the difference in the mean source-guessing parameter $g$ from the chance level of .50 (i.e., $\Delta g-.50$ ). A statistical meaningfully difference of posterior parameter distributions would be reflected in a $\mathrm{BCI}$ of the difference that excluded zero. In the 10-minute condition, source guessing differed substantially from chance-level guessing in Task $1, \Delta(g-.50)=.07[.03, .11]$, and Task $2, \Delta(g$ $-.50)=.06[.02, .09]$, thus replicating prior findings of sourceguessing biases in the domain of age stereotypes (in traditional aggregate-MPT analyses; Kuhlmann et al., 2016). In the 7-day condition, source guessing exceeded .50 only in the first task, $\Delta(g-.50)=.05[.01, .08]$ but not in second task one week later, $\Delta(g-.50)=.01[-.03, .04]$.

\section{Stability}

Relative stability We define relative stability as the cross-task correlations of source-monitoring parameters. These were automatically estimated in the latent-trait approach as implemented in TreeBUGS (Heck et al., 2018). Table 3 presents estimates of the between-task correlations of each sourcemonitoring parameter based on the posterior samples. For source guessing, the individual-level correlation across tasks is also depicted in Fig. 2. Numerically, these source-guessing correlations were in the positive direction, suggesting that participants whose source guessing was biased in the first test also tended to be biased in the second test. However, both correlations were rather small and their BCIs included zero, such that a negative correlation or null population correlation cannot be ruled out. Thus, there was no conclusive evidence for individual-level relative stability of the stereotype bias in source guessing. To exclude poor reliability of source guessing as an explanation for the observed weak cross-task correlations, we confirmed via split-half analyses that source guessing was estimated with satisfactory reliability within each task ( $\hat{\rho}=.47-.70$; see Appendix A for further details). In addition, we conducted simulation analyses to confirm that trait-level

\footnotetext{
${ }^{8}$ The misfit of the covariance structure in the 10-minute condition persisted $(p$ $<.01)$ for all other submodels. The covariance misfit in the 10-minute condition was caused by seven participants for whom Submodel 4 did not fit in the mean structure either. The exclusion of these seven participants led to a significant improvement of both model fit indicators $\left(T_{1}=.349, T_{2}=.614\right)$, without substantially changing the overall parameter group means and correlations. We thus decided to retain Submodel 4 for all further analyses, which has been successfully applied to analyze source-monitoring data in prior studies with similar paradigms (Bayen et al., 2000; Kuhlmann et al., 2016).
} 
Table 2 Multinomial processing tree model group-level parameter estimates of both experiments

\begin{tabular}{|c|c|c|c|c|c|}
\hline \multirow[t]{2}{*}{ Experiment } & \multirow[t]{2}{*}{ Task } & \multicolumn{4}{|l|}{ Parameters } \\
\hline & & $D$ & $d$ & $b$ & $g$ \\
\hline \multicolumn{6}{|l|}{ Experiment 1} \\
\hline \multirow[t]{2}{*}{10 minutes } & 1 & $.73(.40)[.70, .76]$ & $.36(1.46)[.24, .48]$ & $.42(.52)[.36, .47]$ & $.57(.37)[.53, .61]$ \\
\hline & 2 & $.67(.40)[.63, .70]$ & $.38(1.83)[.23, .54]$ & $.37(.59)[.31, .42]$ & $.56(.30)[.52, .59]$ \\
\hline \multirow[t]{2}{*}{7 days } & 1 & $.71(.35)[.69, .74]$ & $.39(1.12)[.28, .49]$ & $.42(.58)[.36, .48]$ & $.55(.36)[.51, .58]$ \\
\hline & 2 & $.69(.40)[.65, .72]$ & $.38(1.35)[.27, .50]$ & $.32(.50)[.27, .36]$ & $.50(.29)[.47, .54]$ \\
\hline \multicolumn{6}{|c|}{ Experiment 2} \\
\hline & 1 & $.55(.29)[.52, .58]$ & $.34(1.01)[.25, .43]$ & $.48(.36)[.44, .51]$ & $.59(.47)[.55, .62]$ \\
\hline & 2 & $.68(.44)[.65, .72]$ & $.53(.71)[.47, .60]$ & $.33(.58)[.28, .38]$ & $.51(.45)[.47, .55]$ \\
\hline
\end{tabular}

Note. We used Submodel 4 of the Two-high-threshold multinomial processing tree model from Bayen et al., (1996) adapted to our paradigm. Parameter values were estimated using the latent-trait Bayesian-hierarchical approach (Klauer, 2010) with the $R$ package TreeBUGS (Heck et al., 2018). See text for further details. Parentheses represent standard deviations (on the probit scale). Brackets indicate $95 \%$ Bayesian credibility intervals $(\mathrm{BCI}) . D=$ probability of recognizing a statement as previously presented and probability of knowing that a distractor statement is new; $d=$ probability of correctly remembering the (either typical or atypical) source of a statement; $b=$ probability of guessing that an unrecognized statement is old; $g=$ probability of guessing that a statement had been presented by the source with the typical age/gender for this statement when the source is not remembered

correlations of .70 between source guessing across tasks could have been recovered with satisfactory precision in the latent-trait model with the given number of items and participants. Crucially note, however, that our recovery simulations, about which more details can be retrieved from the Supplemental Material, showed that weaker correlations (i.e., $\rho=.30$ ) could not be precisely recovered; thus, there may be a true weak correlation of source-guessing bias across tasks, but it is smaller than the traitlike across-task correlation reported for old-new guessing.

Indeed, in contrast to source guessing, other memory and guessing processes showed strong relative stability across time (see Table 3). This also pertained to the item-guessing parameter $b$, which measures the tendency to guess old when an item is not recognized and can be interpreted as the MPT analogue to the old-new response bias in the signal-detection framework underlying Kantner and Lindsay (2012). As is evident in Table 3, we conceptually replicated their findings of cross-time relative stability in item guessing. Additionally, we generally replicated the results from Kantner and Lindsay when fitting the SDT bias measure $c$ for item memory to the data as used by the authors (whereas adapting the bias measure $c$ for source memory did not result in substantial correlations across tasks). A description of the SDT analyses and their results are summarized in Appendix B.

Absolute stability As the correlative evidence with regard to relative stability largely hinges on the observed interindividual variance in source guessing, a restriction of variance as present (at least to some extent) in this first experiment, may mask existing comparable source-guessing parameters on an individual level. For this reason, we further tested whether participants' source-guessing bias remained stable across tasks in absolute terms by sampling the posterior distribution of the absolute mean differences between both tasks. ${ }^{9}$ In the 10 -minute condition, the absolute mean difference in the source-guessing parameter across tasks indicated a change to a credible extent, but it was of negligible size, $\mid \Delta$ (Task 1 - Task 2$) \mid=.02[.001, .06]$. In the 7-day condition, the mean difference in the source-guessing parameter across tasks also changed credibly, albeit the change was again fairly small, $\mid \Delta$ (Task $1-$ Task 2$) \mid=.04[.003, .09]$. That is, as graphically illustrated in Fig. 2, most participants' absolute reliance on stereotypes in source guessing as measured by source-guessing bias was to a fairly comparable extent in both tasks. A closer inspection of the reliance on stereotypes in source guessing revealed that for only $10.53 \%$ (12 out of 114) of participants in the 10-minute condition and $22.73 \%$ ( 25 out of 110) in the 7-day condition, the mean absolute difference was larger than .15 between tasks. That is, albeit some participants changed their guessing behavior to a substantial degree, the vast majority of participants did not-irrespective of the time interval between tasks. Further, the guessing bias was descriptively more pronounced in the first task for most individuals $\left(n_{10}\right.$ minutes $=63,55.26 \%$ of individuals; $n_{7 \text { days }}: 74$, $67.27 \%$ of individuals), indicating regression to the mean tendencies (also evident from Fig. 2). Thus, in contrast to the correlational relative stability analysis, the analysis of the more conservative absolute stability in individual source guessing across time suggested that stereotype reliance in source guessing is stable across time in the majority of participants.

\section{Covariates of source guessing}

Table 4 presents estimates of the correlations between source guessing and $z$-transformed covariates, and Table 5 presents

\footnotetext{
${ }^{9}$ We thank Adam Osth for suggesting this analysis.
} 
Table 3 Across-task correlations between source-monitoring parameters of both experiments

\begin{tabular}{|c|c|c|c|c|c|}
\hline \multirow[t]{2}{*}{ Experiment } & \multirow[t]{2}{*}{ Task 1} & \multicolumn{4}{|l|}{ Task 2} \\
\hline & & $D$ & $d$ & $b$ & $g$ \\
\hline \multicolumn{6}{|l|}{ Experiment 1} \\
\hline \multirow[t]{4}{*}{10 minutes } & $D$ & $.64[.43, .82]$ & $.37[.12, .60]$ & $-.21[-.46, .06]$ & $-.11[-.42, .21]$ \\
\hline & $d$ & $.32[.06, .54]$ & $.31[.07, .52]$ & $-.16[-.41, .10]$ & $-.34[-.61,-.03]$ \\
\hline & $b$ & $-.15[-.42, .14]$ & $-.04[-.33, .25]$ & $.82[.64, .94]$ & $.23[-.10, .55]$ \\
\hline & $g$ & $-.03[-.31, .26]$ & $-.07[-.35, .21]$ & $.14[-.16, .41]$ & $.27[-.06, .56]$ \\
\hline \multirow[t]{4}{*}{7 days } & $D$ & $.45[.19, .69]$ & $-.02[-.30, .26]$ & $-.06[-.36, .25]$ & $-.05[-.37, .27]$ \\
\hline & $d$ & $.31[.04, .54]$ & $.14[-.12, .38]$ & $-.24[-.52, .04]$ & $-.10[-.41, .22]$ \\
\hline & $b$ & $-.08[-.35, .21]$ & $-.10[-.37, .17]$ & $.38[.06, .65]$ & $.03[-.32, .36]$ \\
\hline & $g$ & $-.11[-.38, .17]$ & $-.03[-.32, .26]$ & $.17[-.15, .47]$ & $.20[-.14, .52]$ \\
\hline \multicolumn{6}{|l|}{ Experiment 2} \\
\hline & $D$ & $.70[.50, .85]$ & $.35[.07, .60]$ & $-.13[-.41, .15]$ & $-.23[-.49, .05]$ \\
\hline & $d$ & $.31[.06, .55]$ & $.29[.03, .53]$ & $.06[-.22, .34]$ & $-.06[-.33, .21]$ \\
\hline & $b$ & $-.11[-.36, .15]$ & $.09[-.19, .35]$ & $.78[.61, .91]$ & $-.09[-.34, .18]$ \\
\hline & $g$ & $-.22[-.43, .01]$ & $-.26[-.48,-.02]$ & $.14[-.11, .39]$ & $.31[.07, .53]$ \\
\hline
\end{tabular}

Note. Sampled population correlation coefficients $\hat{\rho}$ are displayed. We used Submodel 4 of the two-high-threshold multinomial processing tree model from Bayen et al. (1996) adapted to our paradigm. Parameter values were estimated using the latent-trait Bayesian-hierarchical approach (Klauer, 2010) with the $R$ package TreeBUGS (Heck et al., 2018). See text for further details. Brackets indicate $95 \%$ Bayesian credibility intervals $(\mathrm{BCI}) . D=$ probability of recognizing a statement as previously presented and probability of knowing that a distractor statement is new; $d=$ probability of correctly remembering the (either typical or atypical) source of a statement; $b=$ probability of guessing that an unrecognized statement is old; $g=$ probability of guessing that a statement had been presented by the source with the typical age/gender for this statement when the source is not remembered. Substantial correlations (BCI excludes 0 ) are marked in boldface

Experiment 1

10 minutes
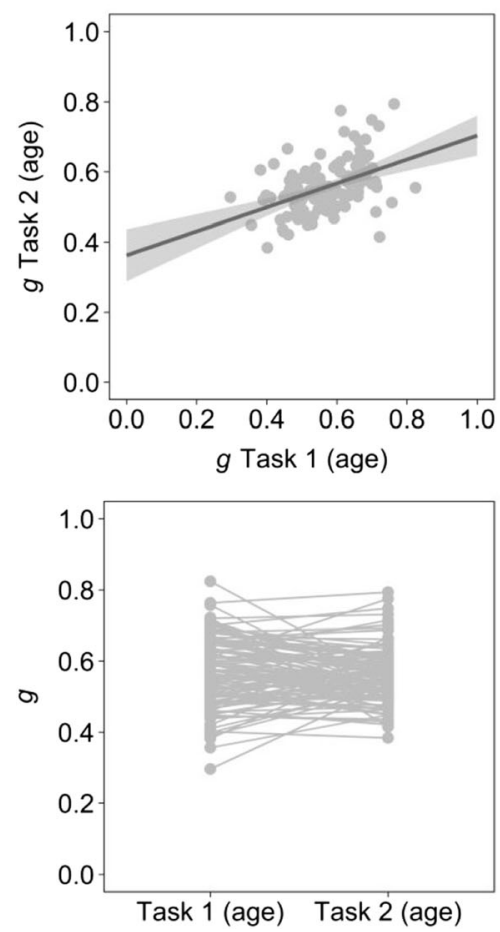

Experiment 1

7 days
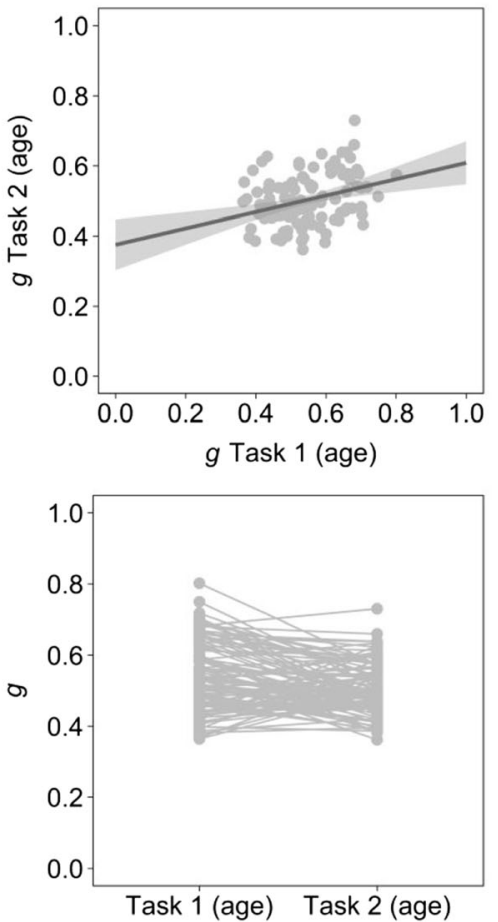

Experiment 2
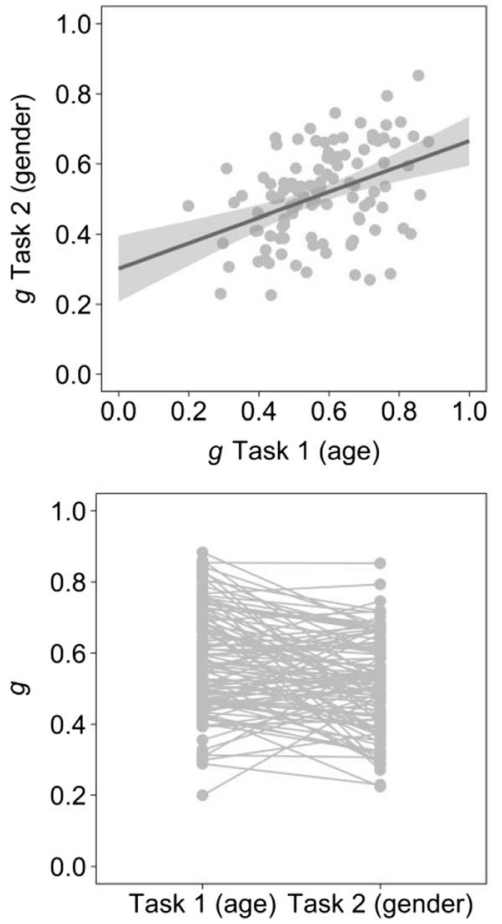

Fig. 2 Top row: Sampled population correlation coefficients $\hat{\rho}$ of sourceguessing parameters $(g)$ across tasks. Individual correlations are displayed in gray points as well as the linear trend line. Error bars represent standard errors (gray). Bottom row: Individual source-guessing parameter estimates connected across tasks. The respective task-specific knowledge domain is displayed in brackets. For both rows, first graph $=$ Experiment 1, 10-minute condition; second graph $=$ Experiment 1,7 -day condition; third graph $=$ Experiment 2 


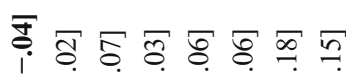
جิ $=0$

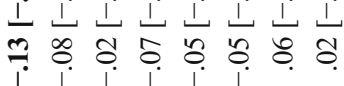

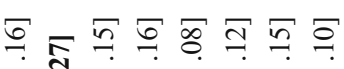
o.

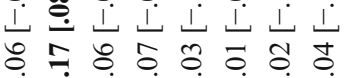

$\varangle$

떠

龸
0

点

甹

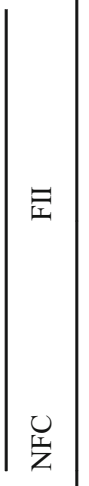

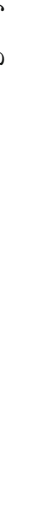

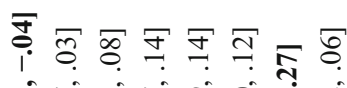

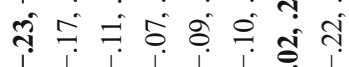

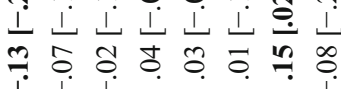

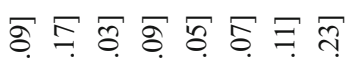
$\Rightarrow$ तो $0=0$

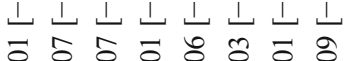

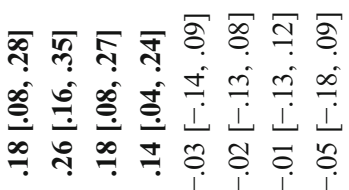

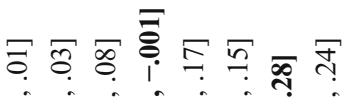

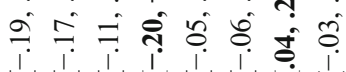

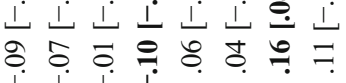

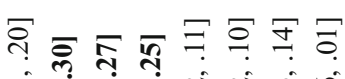
ㅇํㅇำ

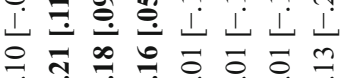

Ð

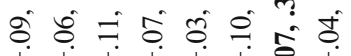

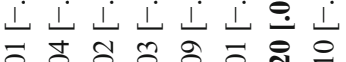<smiles>[13CH3]</smiles>

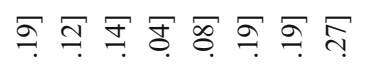

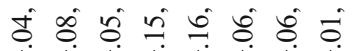

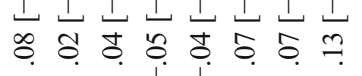

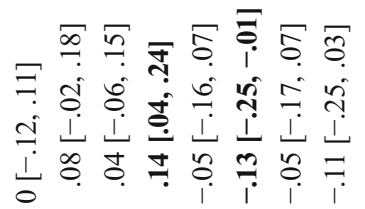

so

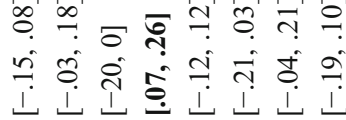
\%

F $\subsetneq 9$

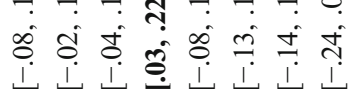
\%

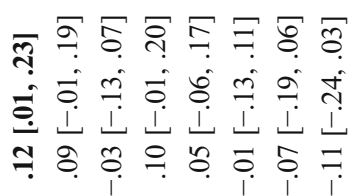

당 คิ $⿻$ की

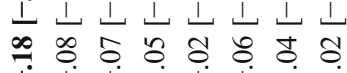

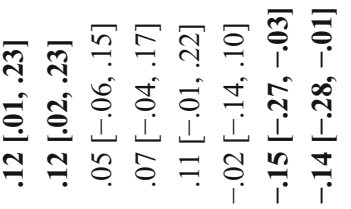

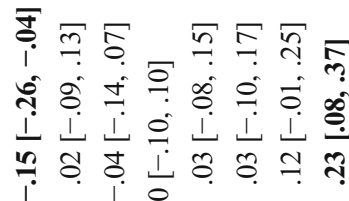

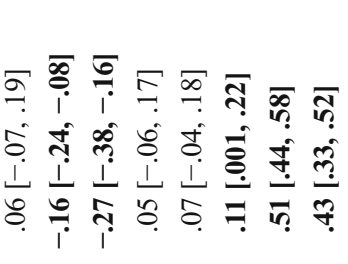

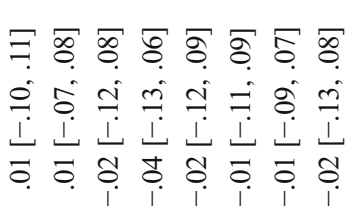

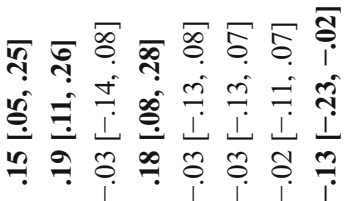

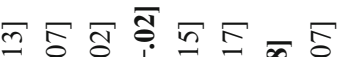

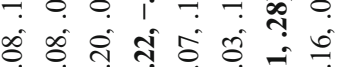

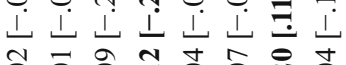

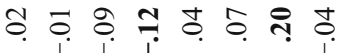

$\infty \bar{\sigma} \infty \Xi \Xi$

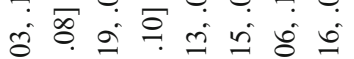

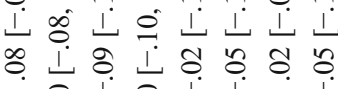

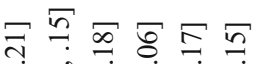

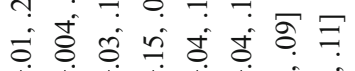

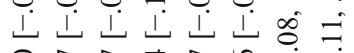

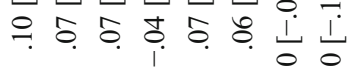

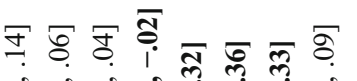

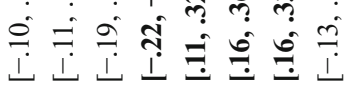

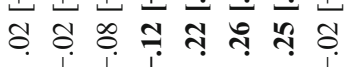

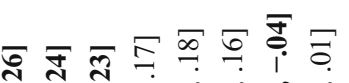

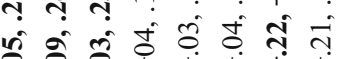

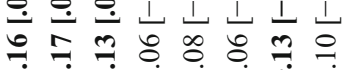
$-2-2-2-2$

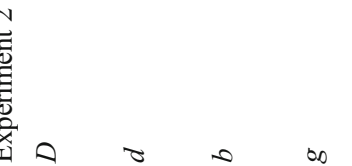

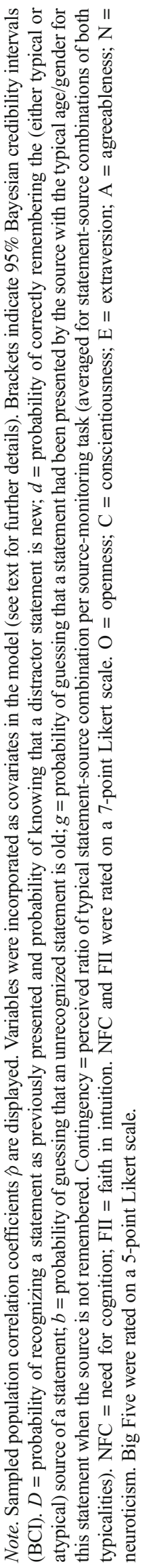


Table 5 Descriptive statistics for the covariates of both experiments

\begin{tabular}{|c|c|c|c|c|c|c|c|c|c|}
\hline \multirow[t]{2}{*}{ Experiment } & \multicolumn{2}{|c|}{ Contingency } & \multicolumn{2}{|c|}{ Cognitive-processing styles } & \multicolumn{5}{|c|}{ Personality traits: Big Five } \\
\hline & & & NFC & FII & $\mathrm{O}$ & $\mathrm{C}$ & $\mathrm{E}$ & $\mathrm{A}$ & $\mathrm{N}$ \\
\hline $\begin{array}{l}\text { Experiment } 1 \\
10 \text { Minutes }\end{array}$ & \multicolumn{8}{|c|}{10 Minutes } & \\
\hline$M$ & .51 & .52 & 4.95 & 4.42 & 3.69 & 3.64 & 3.31 & 3.86 & 2.85 \\
\hline$S D$ & .16 & .16 & .82 & .84 & .66 & .68 & .58 & .58 & .75 \\
\hline Min & .10 & .13 & 3.07 & 1.80 & 1.83 & 1.80 & 2 & 2.50 & 1 \\
\hline $\operatorname{Max}$ & .95 & .90 & 7 & 6.13 & 5 & 5 & 4.33 & 4.83 & 4.83 \\
\hline \multicolumn{10}{|l|}{7 days } \\
\hline M & .53 & .51 & 5.14 & 4.22 & 3.76 & 3.66 & 3.23 & 3.88 & 2.81 \\
\hline$S D$ & .14 & .15 & .72 & .90 & .66 & .65 & .65 & .60 & .67 \\
\hline Min & .15 & .20 & 3.50 & 1.80 & 1.17 & 2.20 & 1.33 & 2 & 1.17 \\
\hline $\operatorname{Max}$ & .88 & .85 & 6.71 & 6.27 & 5 & 5 & 4.50 & 5 & 5 \\
\hline \multicolumn{10}{|l|}{ Experiment 2} \\
\hline$M$ & .52 & .50 & 5.08 & 4.28 & 3.67 & 3.44 & 3.48 & 3.85 & 2.78 \\
\hline$S D$ & .16 & .13 & .77 & .73 & .62 & .54 & .64 & .59 & .75 \\
\hline Min & .17 & .20 & 1.93 & 2 & 2.33 & 2.33 & 1.83 & 1.83 & 1.33 \\
\hline $\operatorname{Max}$ & .92 & .83 & 6.79 & 6.20 & 5 & 4.50 & 4.83 & 5 & 4.67 \\
\hline
\end{tabular}

Note. $\mathrm{NFC}=$ need for cognition; $\mathrm{FII}=$ faith in intuition; $\mathrm{O}=$ openness; $\mathrm{C}=$ conscientiousness; $\mathrm{E}=$ extraversion; $\mathrm{A}=$ agreeableness; $\mathrm{N}=$ neuroticism. NFC and FII were rated on a 7-point Likert scale; Big Five were rated on a 5-point Likert scale. Contingency = perceived ratio of typical statementsource combination per source-monitoring task (averaged for statement-source combinations of both typicalities). $M=$ means, $S D=$ standard deviation, Min = minimum, Max = maximum. All values are aggregated across items

descriptive statistics of these covariates. We found weak but substantial negative correlations between source guessing and NFC for both tasks in the 7-day condition. For all other correlations in both conditions, the $95 \%$ BCIs included zero, revealing that the influence of any Big Five domain and FII on source guessing was unreliable. Thus, we could not identify any systematic personality-trait predictors of stereotype-based source guessing.

Regarding the within-task relationship between source memory and source guessing, we found a negative trend (10 minutes; Task 1: $\hat{\rho}=-.15[-.44, .14]$; Task $2: \hat{\rho}=-.19[-.51$, $.14]$ and 7 days; Task $1: \hat{\rho}=-.29[-.57, .01]$; Task $2: \hat{\rho}=-.28$ $[-.59, .07])$ suggesting that source guessing becomes more stereotype based with poorer source memory (in line with experimental manipulations; Kuhlmann et al., 2016). However, the BCIs of these correlations again included zero. Additionally, the contingency judgment (proportion of typical statement-source combinations in the study phase, averaged for typically-old and typically-young statement-source combinations) for each source-monitoring task was incorporated as a covariate in the model. On the mean level (see Table 5), the contingency judgment was not biased in either condition (i.e., all $p \mathrm{~s} \geq .635$ testing against .50), except for the first task in the 7-day condition, which showed a small overestimation of typical statement-source combinations, $t(109)=2.29, p<$ $.05, B F_{10}=1.27$. Correlations between this contingency judgment and source guessing were numerically in the expected positive direction in all conditions. However, these correlations were only substantial (i.e., BCI excludes zero) in the first task of the 10-minute condition and the second task of the 7-day condition. Thus, we partially replicated prior findings of the probability-matching account of sourceguessing bias (Arnold et al., 2013; Bayen \& Kuhlmann, 2011; Spaniol \& Bayen, 2002), which posits that when people do not remember the source, they match their source-guessing behavior to the perceived contingencies between items and sources.

\section{Experiment 2}

One potential limitation of our first experiment is that participants were faced with the similar (albeit different statements and names) task twice, which could elicit reactance in the second (repeated) task as participants respond to the same type of statements. They may have actively reflected on the first task during the time interval between tasks (albeit being relatively short in the 10-minute condition) and, consequently, changed their response behavior for the second task based on a strategy, or generated an internal hypothesis about the experiment. To test whether the weak relative stability in the first experiment was caused by using the same stereotype domain twice, we conducted a second experiment that specifically tested the effect of item material that reflected distinct knowledge domains: age (analogous to Experiment 1) and 
gender stereotypes. This also allowed us to test whether the absolute stability in source-guessing bias found in Experiment 1 generalizes across different knowledge domains.

\section{Method}

\section{Participants and design}

Participants were 108 students of the University of Mannheim (44\% psychology majors; $71 \%$ women), with a mean age of 21.47 years $(S D=2.25$ years, age range: $18-26$ years). Our sample size considerations and exclusion criteria were identical to those of Experiment 1; we additionally excluded participants of Experiment 1. Again, participants received course credit or monetary compensation.

\section{Material}

Ninety age-stereotypical statements were selected from the same survey study (Kuhlmann et al., 2017) as in Experiment 1 , using a cutoff criterion of $>3.2$ for one age group and $\leq 2.9$ for the other age group. By needing age-stereotypical statements for one task only, we were able to change the item number from 60 to 90 per task compared with Experiment 1 to increase estimation precision.

Gender-stereotypical items for the second sourcemonitoring task consisted of everyday statements that were normed by a subset of 86 participants $(M=21.55$ years, $S D$ $=1.85$ years, age range: $18-26$ years) from Experiment $1 .^{10}$ Statements reflected the domains "career" and "family" for both genders (i.e., men/career, women/career, men/family, women/family). An example statement is: "I see myself as the head of our family" (men/family). Participants either rated the statements' typicality for a man or a woman (randomly assigned; between subjects) on a 5-point Likert scale ( $1=$ very atypical, $5=$ very typical). ${ }^{11}$ We defined a statement as

\footnotetext{
${ }^{10}$ All participants of Experiment 1 completed the gender-typicality rating in Experiment 1 but we prepared and started data collection for Experiment 2 before Experiment 1 was finished.

${ }^{11}$ The original item set consisting of 420 statements was provided by Dr. Marie Luisa Schaper (Heinrich Heine University Düsseldorf). Statements for men and women were generated based on the domains "career" and "family" for both genders (i.e., men/career, women/career, men/family, women/family) and were originally rated on a 5-point scale $(1=$ strongly male, $5=$ strongly female). Ninety-six statements (typically-female statements: $M=3.07, S D=$ .33 ; typically-male statements: $M=3.17, S D=.28,48$ statements from each domain) with equal typicality ratings from male and female participants and 65 additional statements (typically-female statements: $M=3.23, S D=.42$; typically-male statements: $M=3.01, S D=.84 ; 27$ statements from the career domain $\& 38$ statements from the family domain) from the original item pool were chosen for our norming study to have a sufficient large number of typically-male and typically-female statements in our source-monitoring task. To have comparable typicality ratings and cut-off criteria between sourcemonitoring tasks with different material, we adjusted the response scale to a 5-point Likert scale, as used in Experiment 1, ranging from 1 = very atypical to 5 = very typical for the norming in Experiment 1.
}

typically male if the mean typicality rating for a male person was $>3.2$ and, at the same time, $\leq 3$ for a female person, and vice versa for typically-female statements. We randomly selected 90 statements to be equally distributed to the four gender $\times$ domain categories. Statements per category did not differ in terms of their typicality ratings from male and female participants, all $p \mathrm{~s} \geq .50$. Each of the 90 -item sets was divided into three matched subsets of 30 statements, of which two subsets served as study lists and one subset as distractors at test. We used the same source labels (names) as in Experiment 1 for both tasks.

\section{Procedure}

Participants completed both source-monitoring tasks in one session, structured like the first session of Experiment 1's 10-minute condition, with the exception that participants filled out the demographic questionnaire, the BFI and the REI during the 10-minute break. We held task order constant across participants: All participants first completed the task with agestereotypical item material, followed by the task with genderstereotypical item material.

\section{Results and discussion}

\section{Model-based analyses}

As in Experiment 1, we analyzed the data with the 2HTSM Submodel 4 (Bayen et al., 1996) using the Bayesianhierarchical latent-trait approach in TreeBUGS (Heck et al., 2018). We obtained good model fit for both the mean $\left(T_{1}, p\right.$ $=.45)$ and covariance $\left(T_{2}, p=.22\right)$ structure. Group-level estimates of the four parameters are presented in Table 2. On the group level, source guessing exceeded chance level (.50) for the first task, $\Delta(g-.50)=.09[.05, .12]$, but not for the second task using gender stereotypes, $\Delta(g-.50)=.01[-.03$, $.05]$. Participants changed from being biased when performing an age stereotype source-monitoring task to being unbiased in the second source-monitoring task with gender-stereotypical item material 10 minutes later.

\section{Stability}

Relative stability Table 3 presents estimates of the correlations between the four source-monitoring processes across the two tasks. Although the cross-task correlation of source guessing was again rather small, it was substantial (i.e., BCI excludes zero). Thus, participants who guessed based on age stereotypes in the first task, were also more likely to guess based on gender stereotypes in the second task (see also Fig. 2). Albeit the correlation analysis suggested credible relative stability across knowledge domains, the magnitude is again rather small and descriptively comparable to Experiment 1 , in 
which this correlation, however, did not reach statistical credibility. This seemingly inconsistent result across our experiments is to be expected, given our recovery simulations showing that with the given sample and item sizes, only large traitlike cross-task correlations of source guessing could be recovered satisfactorily, whereas there are issues with recovering smaller correlations. Thus, there is some evidence for crosstask relative stability of knowledge reliance in source guessing, but it is less stable than it would be expected for a cognitive trait. Again, within-task split-half correlations indicated good reliability of source guessing across the first task using age stereotypes, $\hat{\rho}=.75[.56, .89]$, and the second task using gender stereotypes, $\hat{\rho}=.70[.45, .88]$ (see Appendix A for further details), such that poor reliability cannot explain the observed weak cross-task correlation.

Other memory and guessing parameters also showed relative stability (see Table 3). Old-new item guessing was highly correlated across tasks, again conceptually replicating Kantner and Lindsay (2012). In addition, we again replicated the results from Kantner and Lindsay with the SDT bias measure $c$ for item memory (whereas adapting the bias measure $c$ for source memory did not result in credible correlations across tasks; see Appendix B).

Absolute stability To account for the observed group-level differences in source guessing between tasks, we calculated the difference between each individual guessing estimate and the respective task group-level mean to relativize each individual's strength of knowledge reliance within each task. We then sampled the absolute mean difference for this meancentered guessing score between the two tasks. The difference $\mid \Delta$ (Task 1 - Task 2)| was .12 $[.002, .36]$-again indicating some changes in source guessing between tasks (see Fig. 2). However, the absolute mean difference was only larger than .15 for $36.11 \%$ of participants (39 out of 108 ). Thus, albeit some participants changed their guessing behavior between tasks, the majority of participants did not and instead showed fairly stable guessing tendencies. As already indicated by diverging group-level guessing biases between tasks, on an individual level, the guessing bias was descriptively more pronounced in the first task for most individuals ( $n=73,67.59 \%$ of individuals), possibly indicating regression to the mean tendencies in the second task. Further, the somewhat larger mean change in source guessing across tasks (and for a somewhat larger proportion of participants) in comparison to Experiment 1 may be due to differential strength of age versus gender stereotypes within participants (albeit matched average stereotype strength of the item material).

\section{Covariates of source guessing}

Estimated correlations between source guessing and $z$-transformed covariates are presented in Table 4 . We found a substantial negative correlation of source guessing with NFC and a substantial positive correlation with FII in the first task. However, this pattern did not emerge for the second task. For all other correlations, the $95 \% \mathrm{BCI}$ included zero showing that the influence of any Big Five domain on source guessing was estimated unreliably. This indicates that a (already rather weak) cognitive trait is not necessarily linked to a personality trait, as suggested by Kantner and Lindsay (2012).

Further, there was a negative correlation between source memory and source guessing for the first task (Task $1: \hat{\rho}=$ $-.48[-.69,-.25]$; Task $2: \hat{\rho}=-.08[-.34, .19])$, showing that poorer source memory is, the more source guessing becomes stereotype based (confirming the trend of Experiment 1 and replicating, e.g., Arnold et al., 2013; Kuhlmann et al., 2016). Participants' contingency judgments at the end of each task showed no bias at all (see Table 5). Participants seemed to perceive the true 50:50 contingency of statement-source combinations almost perfectly, both $p \mathrm{~s} \geq .116$, tested against .50 . As presented in Table 4, the estimated correlation of source guessing with the respective contingency judgment of each task indicated a strong relationship. Beyond the positive trends observed in Experiment 1, the results of Experiment 2 replicated the probability-matching account of sourceguessing bias (Bayen \& Kuhlmann, 2011; Spaniol \& Bayen, 2002; hierarchical MPT: Arnold et al., 2013). People appear to match their source-guessing behavior to the perceived contingencies between items and sources, regardless of the distinct knowledge domain.

\section{General discussion}

In two experiments, we tested the state versus trait-like nature of stereotype-based source guessing. The rationale behind these experiments was derived from previous research documenting intraindividual stability of response bias in item memory and of decision-making processes. Individuals performed two independent study-test cycles of a source-monitoring task. They learned information from two person sources of different ages or gender that needed to be remembered in a later (source) memory test. Information was either typical or atypical for the source's social category (age group or gender). If individuals did not remember, they could have relied on their prior knowledge (i.e., social stereotypes) to infer the source. We examined whether, and if so, to what extent, the reliance on stereotypes is an inherent cognitive trait mirrored in stable guessing behavior across time (Experiment 1) and knowledge domains (Experiment 2). We estimated memory performance and guessing tendencies of each individual with Bayesian-hierarchical MPT models. Cross-task correlations of source guessing revealed (at best) weak positive associations across 10 minutes, seven days, and knowledge domains. In contrast, item guessing (conceptually replicating Kantner \& Lindsay, 2012, 2014, with 
the MPT analogue of their SDT-based item response bias measure) and other source-monitoring processes showed medium to large relative stability across these manipulations. Nonetheless, individual guessing tendencies were mainly stable between tasks when measured in absolute differences. Across experiments, we observed some evidence that the perceived statement-source contingency explained individuals' guessing tendencies, but none of the personality traits and cognitiveprocessing styles consistently did.

\section{Methodological challenges of cross-task relative stability in source guessing}

Although we obtained positive cross-task correlations of source guessing, implying its relative stability, in all experiments and conditions, we acknowledge that these correlations were rather weak and not estimated precisely (i.e., large credibility intervals), resulting in only one substantial cross-task correlation in Experiment 2 (and in Experiment 1's extended sample with $n=164$; see Supplemental Material). However, we caution not to dismiss the relative stability based on these correlational analyses due to several statistical challenges involved in this correlative measure. Although our split-half reliability analyses dismiss poor reliability of source guessing as a methodological explanation, our post hoc parameterrecovery simulations (see Supplemental Material) revealed that only large correlations (i.e., $\rho=.7$ ) could be precisely recovered in the latent-trait 2HTSM Submodel 4 with the given number of observations. Weaker correlations, in particular those of the size observed in both experiments (i.e. $\rho=$ .30 ), could not be recovered with adequate power, particularly because the recovery simulations were based on the smallest number of items and participants in our experiments. The credible cross-task source-guessing correlation for the extended sample indicated that even small correlations should be recovered more precisely with an increasing number of observations. We are thus careful to not overinterpret the overlap of two of three source-guessing cross-task correlation's credibility interval with zero, as evidence against relative stability of source guessing. Nonetheless, if source guessing was a highly stable cognitive trait to a comparable extent as the response bias in recognition memory (Kantner \& Lindsay, 2012, 2014) or the knowledge reliance in the recognition-heuristic use (Michalkiewicz \& Erdfelder, 2016), then we should have detected such large cross-task correlation based on the sample size and item numbers in both experiments. As such, we can conclude that source guessing does not show the high relative stability that one would expect for a cognitive trait.

It is notable that we detected medium to large cross-task correlations between the item response bias parameter $b$, confirming its trait-like status (Kantner \& Lindsay, 2012, 2014) and between other source-monitoring parameters. Compared with these parameters, variance in source guessing tended to be smaller, with the largest variance observed in the source-memory parameter $d$. However, variance in source guessing was overall only slightly smaller than in the item response bias parameter $b$, which consistently showed large cross-task correlations.

Thus, a restriction of inter-individual variance in source guessing is rather implausible as an explanation of why we observed only small source-guessing correlations. In contrast, the sparse amount of information (number of observations) underlying each parameter estimate in the 2HTSM model (Bayen et al., 1996) may explain the difficulty to precisely recover estimates of cross-task correlations in source guessing, specifically, whereas the cross-task correlations of other model parameters (including the item response bias parameter $b$ that was of additional interest to us) could be recovered precisely. Specifically, the dependence of source guessing on various preceding parameters may render the detection of small correlations in this parameter more difficult in the data at hand. In addition, in Experiment 1, in which both source-guessing correlations were not substantial, the covariance structure of the data was overall not explained well by the model.

One potential substantive explanation why relative itemguessing stability did not generalize to source guessing may be that the conceptualization of both cognitive processes differs. Item guessing refers to a conservative versus liberal response bias - individuals inherently diverge from each other with regard to their response criterion. Source guessing refers to responding stereotypically versus atypically when (source) memory is absent. Contrary to item guessing, no task-/material-unrelated criterion such as conservative versus liberal exists for source guessing without prior knowledge activation. Source guessing cannot be investigated and interpreted in a sensible manner without the activation of schema/stereotypes as a conservative or liberal response criterion (which would just mean to respond to one source more often than the other). Although the interpretation of source guessing depends on what the sources stand for, similar findings such as probability matching have been documented across paradigms with diverse schema and stereotype domains (e.g., Bayen \& Kuhlmann, 2011; Kuhlmann et al., 2016; Kuhlmann et al., 2012; Spaniol \& Bayen, 2002; for an overview, see Kuhlmann \& Bayen, 2016). This is why one can expect our results to be generalizable also to other knowledge-based source-guessing biases (which, however, awaits to be tested in future studies).

We are confident that there is not a general problem with estimating relative correlation stability of parameters from the 2HTSM MPT model. Indeed, our supplemental correlational analyses of the SDT response-bias measure $c$, in item and source memory, replicated the latent-trait MPT-model-based results for item guessing (parameter $b$ ) and source guessing (parameter $g$ ), respectively. Thus, our results are independent of the employed memory measurement model (MPT vs. SDT). Regarding item response bias, this means that 
Kantner and Lindsay's $(2012,2014)$ findings can be robustly replicated within the MPT framework, further evidencing the (relative) stability of responding either liberal or conservative with regard to old-new recognition.

\section{The nature of stereotype-based source guessing}

Given the described inherent methodological drawbacks of estimating relative stability in source guessing, we additionally considered absolute stability in our analyses. Crucially, this absolute difference captures minimal changes in guessing tendencies and can thus be defined as an even stricter measure of stability. This measure revealed rather small changes in the knowledge reliance of source guessing within individuals. In particular, these changes were of negligible size $(\leq .04)$ in Experiment 1, in which the reliance on the same stereotypes (age) was assessed in both tasks. The somewhat larger absolute change in source guessing in Experiment 2 was to be expected, as the transition of knowledge domains (age to gender) between tasks may likely still elicit a substantial change in individuals' guessing behavior. Although we matched average stereotype strength between tasks based on the surveys, the age and gender stereotypes may be of different strength within a given individual. Nonetheless, only about a third of participants showed a substantial change of .15 or larger in source guessing between tasks in Experiment 2. Thus, in absolute terms, source guessing was fairly stable across tasks in both experiments.

Both stability measures broadly result in convergent evidence that source guessing encompasses at least to some extent traitlike features. Although correlations were weaker than one would expect for a trait, the majority of participants remained highly stable in absolute terms in their source guessing, thus fulfilling the more conservative stability criterion. In any case, these stability analyses provided a more nuanced picture of the knowledge reliance in guessing tendencies. In particular, the obtained findings for (absolute) stability of source guessing may speak to the underlying process nature - namely, whether it is a rather automatic cognitive process or under conscious control (cf. Bargh, 1994). One could speculate that source guessing may rather be an automatic cognitive process not under conscious control if it is fairly stable across tasks and, more generally, across situations. Contrary to this, prior research suggested that source guessing relies on systematic rather than on heuristic processing (e.g., Bayen \& Kuhlmann, 2011; Ehrenberg \& Klauer, 2005; Spaniol \& Bayen, 2002; for a theoretical overview, see Johnson et al., 1993; Johnson \& Raye, 2000), and in the present experiments there was also some evidence that the perceived item-source contingency influenced source guessing, which seems to rather reflect systematic processing (cf. Kuhlmann et al., 2012). However, as the two presented experiments only provide indirect evidence, future studies should consider to directly manipulate and test the (automatic versus controlled) nature of source guessing.
However, our results clearly demonstrated that the trait-like nature of source guessing is not as pronounced as for other cognitive biases and thereby diverges from other cognitive processes involved in source attributions. As the process of source guessing may be of more complex nature it may not only depend on other cognitive processes such as source memory but also on the perception of contingencies already at encoding of information (e.g., Arnold et al., 2013) and the need to integrate further knowledge (e.g., about social groups). Therefore, the nature of stereotype-based source guessing may not be entirely comparable to, but qualitatively different from, other response biases (e.g., knowledge reliance in the recognition-heuristic use; Michalkiewicz \& Erdfelder, 2016) or decision-making processes (e.g., such as risk seeking; Glöckner \& Pachur, 2012). The potentially more complex nature of source guessing as a cognitive trait may also explain the absence of its link with personality traits, which is present for the previously named cognitive processes.

In addition to noting that the reliance on prior knowledge may often lead to correct source attributions outside the laboratory, one could further speculate that instead of mere knowledge reliance, stereotype-based source guessing reflects a rational metacognitive bias compensating for (perceived) memory differences between sources (cf. Batchelder \& Batchelder, 2008). In particular, knowledge-based source guessing might be a metacognitive compensation strategy for the sometimes found memory deficit for expected sources (i.e., inconsistency effect; e.g., Bell, Buchner, Kroneisen, \& Giang, 2012; Ehrenberg \& Klauer, 2005; Kroneisen \& Bell, 2013; Küppers \& Bayen, 2014). Even though source guessing has been found to reflect rational metacognitive strategies due to item-memory differences (e.g., Kuhlmann \& Touron, 2011; Meiser, Sattler, \& Von Hecker, 2007), Schaper, Kuhlmann, and Bayen (2019) recently demonstrated that knowledge-based source guessing cannot be described as a rational metacognitive bias. In fact, expectancies about the memorability of expected and unexpected item-source combinations did not predict the knowledge reliance (here: schema) in source guessing.

\section{State determinants of source guessing}

The current findings are of particular importance, as age stereotypes become internalized at a young age and then become selfstereotypes at an older age (Levy, 2009; Levy, Slade, Chung, \& Gill, 2015), which under threat can lead to older adults' underperformance in cognitive and physical tasks (see Lamont, Swift, \& Abrams, 2015, for a meta-analytic review). The somewhat stable reliance on stereotypes and schemas in source guessing potentially maintains or even reinforces stereotypes in general, profoundly influencing how we form impressions about others (e.g., Bell et al., 2013) and how we behave (e.g., in a legal decision-making context; Lindsay, 1994, 2014). Therefore, future studies should be inspired by research 
questions on how to nevertheless explore state-like features to identify specific situational determinants that prompt people to rely more or less on prior knowledge, and age stereotypes in particular, in source guessing. Former research has shown that the stereotype reliance in source guessing can be debiased through effective encoding (Arnold et al., 2013; Bayen \& Kuhlmann, 2011; Kuhlmann et al., 2012; Spaniol \& Bayen, 2002) and by informing people about the negative impact of stereotypes in society (Marsh et al., 2006). However, whether these interventions also help to reduce age-stereotype-based source guessing has not yet been determined. In addition, the long-term effects of such interventions on the maintenance of knowledge reliance need to be examined. Future studies could identify additional state moderators of stereotype-based source guessing related, for instance, to the uniqueness and memorability of the statements and the personal relevance of the person's sources and depicted stereotype. Other external factors that could contribute to the knowledge reliance may be the general motivation to participate or strategy use.

In sum, our experiments are a first step towards unpacking the underlying nature of the cognitive process of source guessing. Along with future studies, a better understanding of trait and state characteristics may help to determine who is most prone to stereotype-biased memory and how this bias may be effectively prevented.

Author note Liliane Wulff and Beatrice G. Kuhlmann, Department of Psychology, School of Social Sciences, University of Mannheim, Germany.

This work was supported by the University of Mannheim's Graduate School of Economic and Social Sciences and the research-training group Statistical Modeling in Psychology (GRK 2277), funded by the German Research Foundation (DFG). Parts of this research were presented at the 2018 International Meeting of the Psychonomic Society in Amsterdam, the Netherlands, the 2018 Tagung experimentell arbeitender Psychologen (TeaP) in Marburg, Germany, and the 2017 Doctoral Workshop of Experimental Psychology (ADok) in Ulm, Germany. Data and results for both experiments presented here are available at https://osf.io/mjcve/?view only $=058 \mathrm{c} 26 \mathrm{e} 0508 \mathrm{e} 4 \mathrm{~d} 699020 \mathrm{ffa} 96 \mathrm{db} 276 \mathrm{~cd}$; the materials of the experiments are available from the corresponding author upon request. The experiments were not preregistered.

We thank Daniel Heck for his active support with regard to Bayesian data analysis. We are grateful to Marie Luisa Schaper for providing the statement material used in Experiment 2. We thank Edgar Erdfelder for helpful input on the research idea of this project and Michelle Dörnte, Helena Horn, Alina Kias, Mario Kolb, Franziska Leipold, Rebecca Maurer, Jennifer Panitz, Theresa Pfeiffer, Ronja Reinhardt, and Alin Toja for their assistance with data collection. We thank Jan Rummel for the opportunity to recruit participants in his lab and the student assistants for their help in collecting data at Heidelberg University.

\section{Appendix A}

In order to examine the stability of stereotype reliance source guessing across tasks (time), we first must ensure that we have a reliable and stable measure of source guessing within each source-monitoring task. To test this, we used the split-half method and created two different lists per test phase from each task consisting of 60 test statements each for Experiment 1. We assigned test statements to the lists based on their statement-source typicality and temporal order in which they appeared in the test phase. Specifically, the chronologically first test statement of each statement-source combination (e.g., typically-young statement originally presented by the source "Schmidt") was assigned to the first list, the second statement of each combination was assigned to the second list and so on for all statement-source combinations (3 sources ["old" source vs. "young" source vs. new] $\times 2$ statement typicalities [old vs. young]). Thus, each list contained an equal number of statements from each statement-source combination. We chose this procedure to have comparable lists (i.e., test halves) regarding their content and testing time. We estimated parameters for the two tests lists of each task with the latent-trait approach, including estimation of the correlations of source-guessing probabilities between lists. We obtained a good model fit for the mean but, again, a less satisfactory model fit for the covariance structure (see Table 6).

For Experiment 2, data preprocessing for this split-half analysis was analogous to Experiment 1 except that we excluded the first statement of each statement-source combination due to the odd number of statements per combination in the item set in general ( 90 items in total; six statement-source combinations each containing 15 statements split into seven statements per half).

\section{Appendix B}

\section{Signal detection theory based analyses of relative stability}

We calculated the signal detection theory (SDT) response-bias measure $c$ for old-new item recognition as follows (Macmillan, 1993):

$c=-0.5\left(z_{\text {Hit Rate }}+z_{\text {False-Alarm Rate }}\right)$

Positive values indicate a bias to respond "new" and negative values indicate a bias to respond "old." Before we calculated hits and false-alarm rates, we added .5 to hits and false alarms and 1 to signal and noise trials to correct for perfect hit rates of 1 and absent false-alarm rates of 0 in the data (cf. Hautus, 1995).

We calculated the response-bias measure $c$ for source memory analogously with our conceptualization of hits and false 
alarms in the source attributions following Dodson, Bawa, and Slotnick (2007). That is, we selected only those items that had been recognized as "old" and for which a source attribution was thus made. We conceptualized source memory as following two distributions: one for (recognized) items that were presented with the age-typical source and one for (recognized) items that were presented with the age-atypical source; analogous to the old-item and new-item distributions for item recognition. Positive values indicate a bias to respond the "atypical source," negative values indicate a bias to respond the "typical source." Results for the SDT analysis can be retrieved from Table 7 .
Table 6 Model-fit statistics for split-half reliability analyses of both experiments

\begin{tabular}{llll}
\hline Experiment & List & \multicolumn{2}{l}{ Model-fit indices } \\
\cline { 3 - 4 } & & $T_{1}$ (Mean) & $T_{2}$ (Covariance) \\
\hline Experiment 1 & & & \\
10 minutes & 1 & .305 & 0 \\
& 2 & .345 & .003 \\
7 days & 1 & .521 & .103 \\
& 2 & .292 & .005 \\
Experiment 2 & & & .417 \\
& 1 & .695 & .033 \\
\hline
\end{tabular}

Note. Values represented the respective posterior predictive $p$-value for the mean $\left(T_{1}\right)$ and covariance structure $\left(T_{2}\right)$ of the data (proposed by Klauer, 2010). Good model fit is indicated by $p>.05$

Table 7 Signal-detection theory-based analyses of response bias for item and source memory

\begin{tabular}{|c|c|c|c|c|}
\hline Experiment & Cognitive process & Task & Bias $c$ & Cross-task correlation \\
\hline \multicolumn{5}{|c|}{ Experiment 1} \\
\hline \multirow[t]{2}{*}{10 minutes } & Item memory & $\begin{array}{l}1 \\
2\end{array}$ & $\begin{array}{l}.08(.31) \\
.16(.35)\end{array}$ & $r(114)=.61, p<.001, B F_{10}=3 e^{10}$ \\
\hline & Source memory & $\begin{array}{l}1 \\
2\end{array}$ & $\begin{array}{l}-.13(.35) \\
-.11(.33)\end{array}$ & $r(114)=.14, p=.08, B F_{10}=.32$ \\
\hline \multirow[t]{2}{*}{7 days } & Item memory & $\begin{array}{l}1 \\
2\end{array}$ & $\begin{array}{l}.07(.34) \\
.20(.31)\end{array}$ & $r(110)=.26, p=.003, B F_{10}=8.97$ \\
\hline & Source memory & $\begin{array}{l}1 \\
2\end{array}$ & $\begin{array}{l}-.12(.34) \\
-.004(.33)\end{array}$ & $r(110)=.02, p=.44, B F_{10}=.12$ \\
\hline \multicolumn{5}{|c|}{ Experiment 2} \\
\hline & Item memory & $\begin{array}{l}1 \\
2\end{array}$ & $\begin{array}{l}.03(.27) \\
.20(.33)\end{array}$ & $r(108)=.62, p<.001, B F_{10}=1.28 e^{10}$ \\
\hline & Source memory & $\begin{array}{l}1 \\
2\end{array}$ & $\begin{array}{l}-.21(.42) \\
-.16(.36)\end{array}$ & $r(108)=.22, p=.01, B F_{10}=1.69$ \\
\hline
\end{tabular}

Note. Mean item-memory and source-memory bias values per task. Positive values of item-memory $c$ refer to guessing that an item was "new," negative values of $c$ refer to guessing that an item was "old." Positive values of source-memory $c$ refer to guessing the "atypical" source, negative values of $c$ refer to guessing the "typical" source. Standard deviations are reported in parentheses. Test statistic = Pearson product-moment correlation between bias measures of both tasks per experiment/condition; $p$ values refer to one-tailed testing; Bayes Factors $B F_{10}$ were computed with JASP (JASP Team, 2018) and are interpreted as substantial to strong evidence in favor of the alternative hypothesis $H_{1}$ for item-memory bias and as substantial evidence in favor of the absence of an effect for source-memory bias following Jeffreys (1961) 


\section{References}

Alba, J. W., \& Hasher, L. (1983). Is memory schematic? Psychological Bulletin, 93(2), 203-231. doi:https://doi.org/10.1037/0033-2909.93. 2.203

Arnold, N. R., Bayen, U. J., Kuhlmann, B. G., \& Vaterrodt, B. (2013). Hierarchical modeling of contingency-based source monitoring: A test of the probability-matching account. Psychonomic Bulletin \& Review, 20(2), 326-333. doi:https://doi.org/10.3758/s13423-012$0342-7$

Bargh, J. A. (1994). The four horseman of automaticity: Awareness, intention, efficiency, and control in social cognition. In R. S. Wyer, Jr., \& T. K. Srull (Eds.), Handbook of social cognition: Basic processes; Applications (pp. 1-40). Hillsdale, NJ: Erlbaum. doi: https:// doi.org/10.1007/s00572-005-0022-9

Batchelder, W. H., \& Batchelder, E. (2008). Metacognitive guessing strategies in source monitoring. In J. Dunlosky \& R. A. Bjork (Eds.), Handbook of metamemory and memory (pp. 211-244). New York, NY: Psychology Press.

Batchelder, W. H., \& Riefer, D. M. (1999). Theoretical and empirical review of multinomial process tree modeling. Psychonomic Bulletin \& Review, 6(1), 57-86. doi:https://doi.org/10.3758/ BF03210812

Bayen, U. J., \& Kuhlmann, B. G. (2011). Influences of source-item contingency and schematic knowledge on source monitoring: Tests of the probability-matching account. Journal of Memory and Language, 64(1), 1-17. doi:https://doi.org/10.1016/j.jml.2010.09. 001

Bayen, U. J., Murnane, K., \& Erdfelder, E. (1996). Source discrimination, item detection, and multinomial models of source monitoring. Journal of Experimental Psychology: Learning, Memory, and Cognition, 22(1), 197-215. doi:https://doi.org/10.1037/0278-7393. 22.1.197

Bayen, U. J., Nakamura, G V, Dupuis, S. E., \& Yang, C.-L. (2000). The use of schematic knowledge about sources in source-monitoring. Memory \& Cognition, 28(3), 480-500. doi:https://doi.org/10.3758/ BF03198562

Bell, R., Buchner, A., Kroneisen, M., \& Giang, T. (2012). On the flexibility of social source memory: A test of the emotional incongruity hypothesis. Journal of Experimental Psychology: Learning, Memory, and Cognition, 38(6), 1512-1529. doi: https://doi.org/10. 1037/a0028219

Bell, R., Giang, T., Mund, I., \& Buchner, A. (2013). Memory for reputational trait information: Is social-emotional information processing less flexible in old age? Psychology and Aging, 28(4), 984-995. doi: https://doi.org/10.1037/a0034266

Bell, R., Mieth, L., \& Buchner, A. (2017). Emotional memory: No source memory without old-new recognition. Emotion, 17(1), 120-130. doi:https://doi.org/10.1037/emo0000211

Cacioppo, J. T., \& Petty, R. E. (1982). The need for cognition. Journal of Personality and Social Psychology, 42(1), 116-131. doi:https://doi. org/10.1037/0022-3514.42.1.116.

Carter, J. D., Hall, J. A., Carney, D. R., \& Rosip, J. C. (2006). Individual differences in the acceptance of stereotyping. Journal of Research in Personality, 40(6), 1103-1118. doi:https://doi.org/10.1016/j.jrp. 2005.11.005

Carver, C. S., \& White, T. L. (1994). Behavioral inhibition, behavioral activation, and affective responses to impending reward and punishment: The BIS/BAS Scales. Journal of Personality and Social Psychology, 67(2), 319-333. doi:https://doi.org/10.1037/00223514.67.2.319

Crawford, M. T., \& Skowronski, J. J. (1998). When motivated thought leads to heightened bias: High need for cognition can enhance the impact of stereotypes on memory. Personality and Social
Psychology Bulletin, 24(10), 1075-1088. doi:https://doi.org/10. 1177/01461672982410005

Danner, D., Rammstedt, B., Bluemke, M., Treiber, L., Berres, S., Soto, C., \& John, O. (2016). Die deutsche Version des Big Five Inventory 2 (BFI-2) [The German version of the Big Five Inventory 2]. Zusammenstellung Sozialwissenschaftlicher Items Und Skalen. doi:https://doi.org/10.6102/zis247

Dodson, C. S., Bawa, S., \& Slotnick, S. D. (2007). Aging, source memory, and misrecollections. Journal of Experimental Psychology: Learning, Memory, and Cognition, 33(1), 169-181. doi:https://doi. org/10.1037/0278-7393.33.1.169

Ehrenberg, K., \& Klauer, K. C. (2005). Flexible use of source information: Processing components of the inconsistency effect in person memory. Journal of Experimental Social Psychology, 41(4), 369387. doi:https://doi.org/10.1016/j.jesp.2004.08.001

Epstein, S., Pacini, R., Denes-Raj, V., \& Heier, H. (1996). Individual differences in intuitive-experiential and analytical-rational thinking styles. Journal of Personality and Social Psychology, 71(2), 390 405. doi:https://doi.org/10.1037/0022-3514.71.2.390

Faul, F., Erdfelder, E., Lang, A. G., \& Buchner, A. (2007). G*Power 3: A flexible statistical power analysis program for the social, behavioral, and biomedical sciences. Behavior Research Methods, 39(2), 175191. doi:https://doi.org/10.3758/BF03193146

Fiske, S. T. (1989). Examining the role of intent: Toward understanding its role in stereotyping and prejudice. In J. S. Uleman \& J. A. Bargh (Eds.), Unintended thought (pp. 253-283). New York, NY: Guilford Press.

Flynn, F. J. (2005). Having an open mind: The impact of openness to experience on interracial attitudes and impression formation. Journal of Personality and Social Psychology, 88(5), 816-826. doi:https://doi.org/10.1037/0022-3514.88.5.816

Gelman, A., \& Rubin, D. B. (1992). Inference from iterative simulation using multiple sequences. Statistical Science, 7(4), 457-472. doi: https://doi.org/10.1214/ss/1177011136

Gilbert, D. T., \& Hixon, J. G. (1991). The trouble of thinking: Activation and application of stereotypic beliefs. Journal of Personality and Social Psychology, 60(4), 509-517. doi:https://doi.org/10.1037/ 0022-3514.60.4.509

Glöckner, A., \& Pachur, T. (2012). Cognitive models of risky choice: Parameter stability and predictive accuracy of prospect theory. Cognition, 123(1), 21-32. doi:https://doi.org/10.1016/j.cognition. 2011.12.002

Goldberg, L. R. (1993). The structure of phenotypic personality traits. American Psychologist, 48(1), 26-34. doi:https://doi.org/10.1037/ 0003-066X.48.1.26

Goldstein, D. G., \& Gigerenzer, G. (2002). Models of ecological rationality: The recognition heuristic. Psychological Review, 109(1), 7590. doi:https://doi.org/10.1093/acprof:oso/9780199744282.003. 0003

Hautus, M. J. (1995). Corrections for extreme proportions and their biasing effects on estimated values of $d^{\prime}$. Behavior Research Methods, Instruments \& Computers, 27(1), 46-51. doi:https://doi.org/10. 3758/BF03203619

Heck, D. W., Arnold, N. R., \& Arnold, D. (2018). TreeBUGS: An R package for hierarchical multinomial-processing-tree modeling. Behavior Research Methods, 50(1), 264-284. doi:https://doi.org/ 10.3758/s13428-017-0869-7

Hicks, J. L., \& Cockman, D. W. (2003). The effect of general knowledge on source memory and decision processes. Journal of Memory and Language, 48(3), 489-501. doi:https://doi.org/10.1016/S0749596X(02)00537-5

Hu, X., \& Batchelder, W. H. (1994). The statistical analysis of general processing tree models with the EM algorithm. Psychometrika, 59(1), 21-47. doi:https://doi.org/10.1007/BF02294263

JASP Team. (2018). JASP (Version 0.10.0) [Computer software]. Retrieved from https://jasp-stats.org/ 
Jeffreys, H. (1961). Theory of probability (3rd ed.). Oxford, England: Oxford University Press.

John, O. P., \& Srivastava, S. (1999). The Big Five Trait taxonomy: History, measurement, and theoretical perspectives. In L. A. Pervin \& O. P. John (Eds.), Handbook of personality: Theory and research (2nd ed., pp. 102-138). New York, NY: Guilford Press.

Johnson, M. K., Hashtroudi, S., \& Lindsay, D. S. (1993). Source monitoring. Psychological Bulletin, 114(1), 3-28. doi:https://doi.org/10. 1037/0033-2909.114.1.3

Johnson, M. K., \& Raye, C. L. (2000). Cognitive and brain mechanisms of false memories and beliefs. In D. L. Schacter \& E. Scarry (Eds.), Memory, brain, and belief (pp. 35-86). Cambridge, MA: Harvard University Press.

Kantner, J., \& Lindsay, D. S. (2012). Response bias in recognition memory as a cognitive trait. Memory \& Cognition, 40, 1163-1177. doi: https://doi.org/10.3758/s13421-012-0226-0

Kantner, J., \& Lindsay, D. S. (2014). Cross-situational consistency in recognition memory response bias. Psychonomic Bulletin \& Review, 21, 1272-1280. doi:https://doi.org/10.3758/s13423-0140608-3

Keller, J., Bohner, G., \& Erb, H.-P. (2000). Intuitive und heuristische Urteilsbildung-verschiedene Prozesse ? Präsentation einer deutschen Fassung des "Rational-Experiential Inventory" sowie neuer Selbstberichtskalen zur Heuristiknutzung [Intuitive and heuristic judgment - different processes? Presentation of a German version of the Rational-Experiential Inventory and of new self-report scales of heuristic use]. Zeitschrift für Sozialpsychologie, 31(2), 87101. doi:https://doi.org/10.1024//0044-3514.31.2.87

Klauer, K. C. (2010). Hierarchical multinomial processing tree modelsA latent-trait approach. Psychometrika, 75(1), 70-98. doi: https:// doi.org/10.1007/s11336-009-9141-0

Klauer, K. C., \& Kellen, D. (2010). Toward a complete decision model of item and source recognition: A discrete-state approach. Psychonomic Bulletin \& Review, 17(4), 465-478. doi:https://doi. org/10.3758/PBR.17.4.465

Kroneisen, M., \& Bell, R. (2013). Sex, cheating, and disgust: Enhanced source memory for trait information that violates gender stereotypes. Memory, 21(2), 167-181. doi:https://doi.org/10.1080/09658211. 2012.713971

Kuhlmann, B. G., \& Bayen, U. J. (2016). Metacognitive aspects of source monitoring. In J. Dunlosky \& S. K. Tauber (Eds.), The Oxford handbook of metamemory (pp. 149-168). New York, NY: Oxford University Press.

Kuhlmann, B. G., Bayen, U. J., Meuser, K., \& Kornadt, A. E. (2016). The impact of age stereotypes on source monitoring in younger and older adults. Psychology and Aging, 31(8), 875-889. doi:https://doi.org/ 10.1037/pag0000140

Kuhlmann, B. G., Kornadt, A. E., Bayen, U. J., Meuser, K., \& Wulff, L. (2017). Multidimensionality of younger and older adults' age stereotypes: The interaction of life domain and adjective dimension. Journals of Gerontology, Series B: Psychological Sciences and Social Sciences, 72(3), 436-440. doi:https://doi.org/10.1093/ geronb/gbv049

Kuhlmann, B. G., \& Touron, D. R. (2011). Older adults' use of metacognitive knowledge in source monitoring: Spared monitoring but impaired control. Psychology and Aging, 26(1), 143-149. doi: https://doi.org/10.1037/a0021055

Kuhlmann, B. G., Vaterrodt, B., \& Bayen, U. J. (2012). Schema bias in source monitoring varies with encoding conditions: Support for a probability-matching account. Journal of Experimental Psychology: Learning, Memory, and Cognition, 38(5), 1365-1376. doi: https:// doi.org/10.1037/a0028147

Küppers, V., \& Bayen, U. J. (2014). Inconsistency effects in source memory and compensatory schema-consistent guessing. Quarterly Journal of Experimental Psychology, 67(10), 2042-2059. doi: https://doi.org/10.1080/17470218.2014.904914
Lamont, R. A., Swift, H. J., \& Abrams, D. (2015). A review and metaanalysis of age-based stereotype threat: Negative stereotypes, not facts, do the damage. Psychology and Aging, 30(1), 180-193. doi: https://doi.org/10.1037/a0038586

Levy, B. R. (2009). Stereotype embodiment: A psychosocial approach to aging. Current Directions in Psychological Science, 18(6), 332336. doi:https://doi.org/10.1111/j.1467-8721.2009.01662.x

Levy, B. R., Slade, M. D., Chung, P. H., \& Gill, T. M. (2015). Resiliency over time of elders' age stereotypes after encountering stressful events. Journals of Gerontology, Series B: Psychological Sciences and Social Sciences, 70(6), 886-890. doi:https://doi.org/10.1093/ geronb/gbu082

Lindsay, D. S. (1994). Memory source monitoring and eyewitness testimony. In D. F. Ross, J. D. Read, \& M. P. Toglia (Eds.), Adult eyewitness testimony: Current trends and developments (pp. 2755). New York, NY: Cambridge University Press. doi: https://doi. org/10.1017/CBO9780511759192.003

Lindsay, D. S. (2014). Memory source monitoring applied. In T. Perfect and D. S. Lindsay (Eds.), The SAGE handbook of applied memory (pp. 59-75). London, UK: Sage. doi: https://doi.org/10.4135/ 9781446294703.n4

Macmillan, N. A. (1993). Signal detection theory as data analysis method and psychological decision model. In G. Keren \& C. Lewis (Eds.), A handbook for data analysis in the behavioral sciences: Methodological issues (pp. 21-57). Hillsdale, NJ: Erlbaum.

Macrae, C. N., Milne, A. B., \& Bodenhausen, G. V. (1994). Stereotypes as energy-saving devices: A peek inside the cognitive toolbox. Journal of Personality and Social Psychology, 66(1), 37-47. doi: https://doi.org/10.1037/0022-3514.66.1.37

Malejka, S., \& Bröder, A. (2016). No source memory for unrecognized items when implicit feedback is avoided. Memory \& Cognition, 44(1), 63-72. doi:https://doi.org/10.3758/s13421-015-0549-8

Marsh, R. L., Cook, G. I., \& Hicks, J. L. (2006). Gender and orientation stereotypes bias source-monitoring attributions. Memory, 14(2), 148-160. doi:https://doi.org/10.1080/09658210544000015

Mathôt, S., Schreij, D., \& Theeuwes, J. (2012). OpenSesame: An opensource, graphical experiment builder for the social sciences. Behavior Research Methods, 44(2), 314-324. doi:https://doi.org/ 10.3758/s13428-011-0168-7

Matzke, D., Dolan, C. V., Batchelder, W. H., \& Wagenmakers, E. J. (2015). Bayesian estimation of multinomial processing tree models with heterogeneity in participants and items. Psychometrika, 80(1), 205-235. doi:https://doi.org/10.1007/s11336-013-9374-9

McCrae, R. R., \& Costa, P. T., Jr. (2008). Empirical and theoretical status of the five-factor model of personality traits. In G. Boyle, G. Matthews, \& D. H. Saklofske (Eds.), The SAGE handbook of personality theory and assessment (pp. 273-294). Thousand Oaks, CA: SAGE Publications.

Meiser, T., Sattler, C., \& Von Hecker, U. (2007). Metacognitive inferences in source memory judgements: The role of perceived differences in item recognition. The Quarterly Journal of Experimental Psychology, 60(7), 1015-1040. doi:https://doi.org/10.1080/ 17470210600875215

Michalkiewicz, M., \& Erdfelder, E. (2016). Individual differences in use of the recognition heuristic are stable across time, choice objects, domains, and presentation formats. Memory \& Cognition, 44(3), 454-468. doi:https://doi.org/10.3758/s13421-015-0567-6

Michalkiewicz, M., Minich, B., \& Erdfelder, E. (2019). Explaining individual differences in fast-and-frugal decision making: Need for cognition, faith in intuition, and the recognition heuristic. Manuscript under review.

Mitchell, K., \& Johnson, M. (2000). Source monitoring: Attributing mental experiences. In E. Tulving \& F. I. M. Craik (Eds.), The Oxford handbook of memory (pp. 179-195). New York, NY: Oxford University Press. 
Perlini, A. H., \& Hansen, S. D. (2001). Moderating effects of need for cognition on attractiveness stereotyping. Social Behavior and Personality: An International Journal, 29(4), 313-321. doi:https:// doi.org/10.2224/sbp.2001.29.4.313

Rammstedt, B., Danner, D., Soto, C. J. \& John, O. P. (2018). Validation of the short and extra-short forms of the Big Five Inventory-2 (BFI-2) and their German adaptations. European Journal of Psychological Assessment. doi:https://doi.org/10.1027/1015-5759/a000481

Roberts, B. W. (2009). Back to the future: Personality and assessment and personality development. Journal of Research in Personality, 43(2), 137-145. doi: https://doi.org/10.1016/j.jrp.2008.12.015

Schaper, M. L., Kuhlmann, B. G., \& Bayen, U. J. (2019). Metamemory expectancy illusion and schema-consistent guessing in source monitoring. Journal of Experimental Psychology: Learning, Memory, and Cognition, 45(3), 470-496. doi: https://doi.org/10.1037/ $\mathrm{x} \operatorname{lm} 0000602$

Sherman, J. W., \& Bessenoff, G. R. (1999). Stereotypes as sourcemonitoring cues: On the interaction between episodic and semantic memory. Psychological Science, 10(2), 106-110. doi:https://doi.org/ 10.1111/1467-9280.00116

Smith, J. B., \& Batchelder, W. H. (2010). Beta-MPT: Multinomial processing tree models for addressing individual differences. Journal of Mathematical Psychology, 54(1), 167-183. doi:https://doi.org/10. 1016/j.jmp.2009.06.007
Soto, C. J., \& John, O. P. (2017). Short and extra-short forms of the Big Five Inventory-2: The BFI-2-S and BFI-2-XS. Journal of Research in Personality, 68, 69-81. doi:https://doi.org/10.1016/j.jrp.2017.02. 004

Spaniol, J., \& Bayen, U. J. (2002). When is schematic knowledge used in source monitoring?. Journal of Experimental Psychology: Learning, Memory, and Cognition, 28(4), 631-651. doi: https:// doi.org/10.1037//0278-7393.28.4.631

Spiegelhalter, D. J., Best, N. G., Carlin, B. P., \& Van Der Linde, A. (2002). Bayesian measures of model complexity and fit. Journal of the Royal Statistical Society: Series B (Statistical Methodology), 64(4), 583-639. doi: https://doi.org/10.1111/1467-9868.00353

Stroebe, W., \& Insko, C. A. (1989). Stereotype, prejudice, and discrimination: Changing conceptions in theory and research. In D. Bar-Tal, C. F. Graumann, A. W. Kruglanski, \& W. Stroebe (Eds.), Stereotyping and prejudice: Changing conceptions (pp. 3-34). New York, NY: Springer. doi: https://doi.org/10.1007/978-1-46123582-8_1

Publisher's note Springer Nature remains neutral with regard to jurisdictional claims in published maps and institutional affiliations. 